\title{
Particle reinforced thermoplastic foams under quasi-static compression
}

\author{
Shunze $\mathrm{Cao}^{1}$, Tao Liu ${ }^{1,2 *}$, Arthur Jones ${ }^{2}$, Walid Tizani ${ }^{1}$ \\ ${ }^{1}$ Centre for Structural Engineering and Informatics \\ ${ }^{2}$ Composites Research Group \\ Faculty of Engineering, University of Nottingham, \\ University Park, Nottingham, NG7 2RD, U.K. \\ Email: Tao.Liu@ @ottingham.ac.uk, Tel: + 44 (0)115 7484059 \\ Date of Submission: 18/02/2019
}

\begin{abstract}
Polymer foams are widely used as the core materials for lightweight sandwich structures. Particle reinforcement can be added into polymer foams to enhance their mechanical properties. In this study, particle reinforced foams were manufactured from Linear Low-Density Polyethylene (LLDPE) powder and cellular ceramic particles. Quasi-static compressive responses of the particles reinforced LLDPE foams were measured at selected volume fractions of polymer and particle reinforcement. The experimental measurements have revealed that the reinforcement enhances the elastic modulus, the yield strength and the energy absorption capacity. Analytical predictions of Young's moduli and yield strengths were obtained based on existing theoretical models to interpret the experimental measurement. Finite element (FE) simulations were conducted to understand the reinforcing mechanisms. The FE model was able to predict the measured compressive responses of the foam samples, which captured the complex interaction between particle reinforcements and the hollow cells of the foams. During the loading, the foam matrix around the particle reinforcement is densified or close to being densified while the foam matrix around hollow spherical cells remains undensified, which suggests that hollow spherical cells are stabilised by particle reinforcements. The studies of the size effects of particle reinforcements and hollow cells suggest that the simulation results were not very sensitive to the selected ranges of diameters.
\end{abstract}

Keywords: Particle reinforced foams; quasi-static compression; Finite element modelling; reinforcing mechanism; energy absorption 


\section{Introduction}

Structural foams have been widely used in the packaging, construction, aerospace and automotive industries owing to lightweight and good performance in energy absorption. (Gibson and Ashby, 1997; Marvi-Mashhadi et al., 2010; Srinivasa and Kulachenko, 2015; Marvi-Mashhadi et al., 2018). To enhance the mechanical properties of foam materials, reinforcements, such as carbon nanotubes (Zegeye and Woldesenbet, 2012), metallic pins (Rice et al., 2006), short fibres and particles (Gupta et al., 2013) have been applied for strengthening purposes. Compared to other reinforcing methods, particle based reinforcement has the advantages of low cost and suitability for large scale production. The uniaxial compressive responses of different types of particle reinforced foams have been reported by researchers. Jayavardhan and Doddamani (2018) studied the uniaxial quasi-static compression of glass micro-balloon reinforced high-density polyethylene (HDPE) foams at strain rates of $0.001-0.1 \mathrm{~s}^{-1}$. They reported that the elastic modulus and yield strength of reinforced foams were higher than those of the neat foams, and the energy absorption capability increased with increasing strain rates. Brown et al. (2011) numerically investigated the quasi-static and dynamic compressions of cellular glass particle reinforced linear low density polyethylene (LLDPE) foams. They reported that, with increasing the volume fraction of glass particles, the yield strength increased and the shock wave velocity decreased. Ceramic particles have been used as the reinforcement to modify the performance of foam materials under uniaxial quasi-static compression (Li et al, 2007; Wichianrat et al., 2012; Esmaeelzadeh et al., 2006; Liu et al., 2009). However, there are no consistent conclusions regarding the reinforcing effects. Li et al. (2007) incorporated ceramic particles of $\mathrm{B}_{4} \mathrm{C}, \mathrm{CBN}$ and $\mathrm{SiC}$ into aluminium foams and found that the elastic modulus, peak stress and energy absorption increase after reinforcing. Wichianrat et al. (2012) produced composite foams using AC3A aluminiun alloy with varying contents (1\%-5\% in mass fraction) of $\mathrm{SiC}$ particles. They found that higher mass fraction of ceramic particle leaded to higher energy absorption capability and higher compressive strength. However, Esmaeelzadeh et al. (2006) demonstrated that the energy absorption efficiency of the SiC particle reinforced AlSi7 closed-cell foam was less than that of neat AlSi7 foam. Liu et al. (2009) investigated the compressive behaviour of 
$\mathrm{SiC}$ reinforced $\mathrm{Zn}-22 \mathrm{Al}$ closed-cell foams. They reported that the ceramic particles reinforced foam became more brittle and the energy absorption efficiency degraded in comparison to nonreinforced foams under compression. Although existing researches have suggested that the porous structures of foams could be influenced by ceramic particle reinforcements, the modification (reinforcing or degradation) mechanism remains elusive. This paper aims to investigate the reinforcing mechanism of particle reinforcements considering the interactions between particle reinforcements and the porous internal structures of foams.

To predict the mechanical properties of particle reinforced foams, numerical models have been created through randomly inserting spherical particles (particle reinforcement) into homogeneous host matrix (foams) without detailed modelling of the porous structures of foams (Yu et al., 2016; Chawla and Chawla, 2006; Brown et al., 2011; Cho et al., 2017). Brown et al. (2011) created a two-dimensional (2D) finite element (FE) model to study the shock wave propagation of cellular glass particle reinforced foam under dynamic compression. The glass particles of a specified size range $(0.5 \mathrm{~mm}-2 \mathrm{~mm}$ diameter) were randomly inserted into a foam matrix domain ( $5 \mathrm{~mm} \times 5 \mathrm{~mm}$ square). Cho et al. (2017) developed a three-dimensional FE model to study the tensile deformation of the hollow glass microspheres reinforced iron syntactic foam. The microspheres were inserted into a homogeneous host matrix by generating the central points of the spheres randomly within a cubic domain. The numerical predictions suggested that the yielding of the foam occurred at the onset of cracking of glass particles, which could not be observed from experiments. For particle reinforced foams, the complex interactions between the particles and the porous structures of the foams were found to play important roles in the mechanical behaviours of the materials. Hence, it might be an oversimplification to treat foams as homogeneous host matrices without detailed modelling of the porous structure. In this study, both particle reinforcements and porous structures of foams were simulated explicitly via 3D finite element models to capture their interactions.

The purpose of this paper is to investigate the reinforcing effects and reinforcing mechanisms with consideration of the interactions between ceramic particle reinforcements and the porous internal structures of foams. The outline of this paper is as follows. The mechanical properties of constituent materials, manufacturing method and characteristics of the porous structure are 
reported in Section 2; the experimental protocol is described in Section 3; the numerical modelling on particle reinforced foams are reported in Section 4; the results obtained by the experimental measurements and finite element predictions are compared and discussed in Section 5.

\section{Materials and manufacturing}

The thermoplastic foams investigated in the paper were made from Linear Low-Density Polyethylene $^{1}$ (LLDPE) powder. The LLDPE powder has a density of $\rho_{s}=0.935 \mathrm{~g} / \mathrm{cm}^{3}$, elastic modulus of $E_{s}=450 \mathrm{MPa}$ and yield strength of $\sigma_{s}=27.5 \mathrm{MPa}$. It is noted that the aim of this research is to investigate the reinforcing mechanism resulting from the interactions between particle reinforcements and porous structures of foams rather than seeking the optimal parent material for the particle reinforcements. The experiments were conducted using lightweight cellular ceramic particles (Maifan natural ceramic stone ${ }^{2}$ ) for reinforcement. The ceramic particles have a density of $\rho_{p}=1.6 \mathrm{~g} / \mathrm{cm}^{3}$ and diameter, $d_{p}$, ranging from $1.412 \mathrm{~mm}$ to $1.955 \mathrm{~mm}$, which follows the normal distribution $d_{p} \sim N\left(d_{p m}=1.7 \mathrm{~mm}, \gamma_{p m}^{2}=0.095 \mathrm{~mm}^{2}\right)$, with $d_{p m}$ representing the mean diameter of particles and $\gamma_{p m}$ the standard deviation. Figure 1 shows the compressive force $\left(P_{E}\right)$-displacement $(\Delta)$ curve obtained from a single particle quasi-static compression test with particle diameter $d_{p}=1.7 \mathrm{~mm}$. Curve fitting via finite element simulations on the single particle compression test indicates that the compressive strength of the parent material of the ceramic particles is $\sigma_{p} \approx 27 \mathrm{MPa}$ (see Section 3.3 for the Finite Element modelling). Assuming elastic Hertzian contacts between the single ceramic particle and the test machine (Samimi et al. 2005; Thornton and Ning, 1998), the effective Young's modulus of the ceramic particles can be estimated as $E_{p}=1.12 \mathrm{GPa}$, using the relationship of

$$
E_{p}=\frac{4 d_{p}^{0.5}}{3 P_{E}\left(1-v_{p}\right)}\left(\frac{\Delta}{2}\right)^{1.5}
$$

where $v_{p}$ denotes the Poisson's ratio of ceramic particles, $v_{p}=0.3$. Both stiffness and strength

${ }^{1}$ A. Schulman, Inc, Ltd, UK.

${ }^{2}$ Qingdao Fujing Group Co. Ltd, Shandong China. 
of the particles are high enough to reinforce low or medium density LLDPE foams that have Young's moduli within the range of $36.95 \mathrm{MPa}$ to $111.64 \mathrm{MPa}$ and yield strength within the range of $1.049 \mathrm{MPa}$ to $3.884 \mathrm{MPa}$.

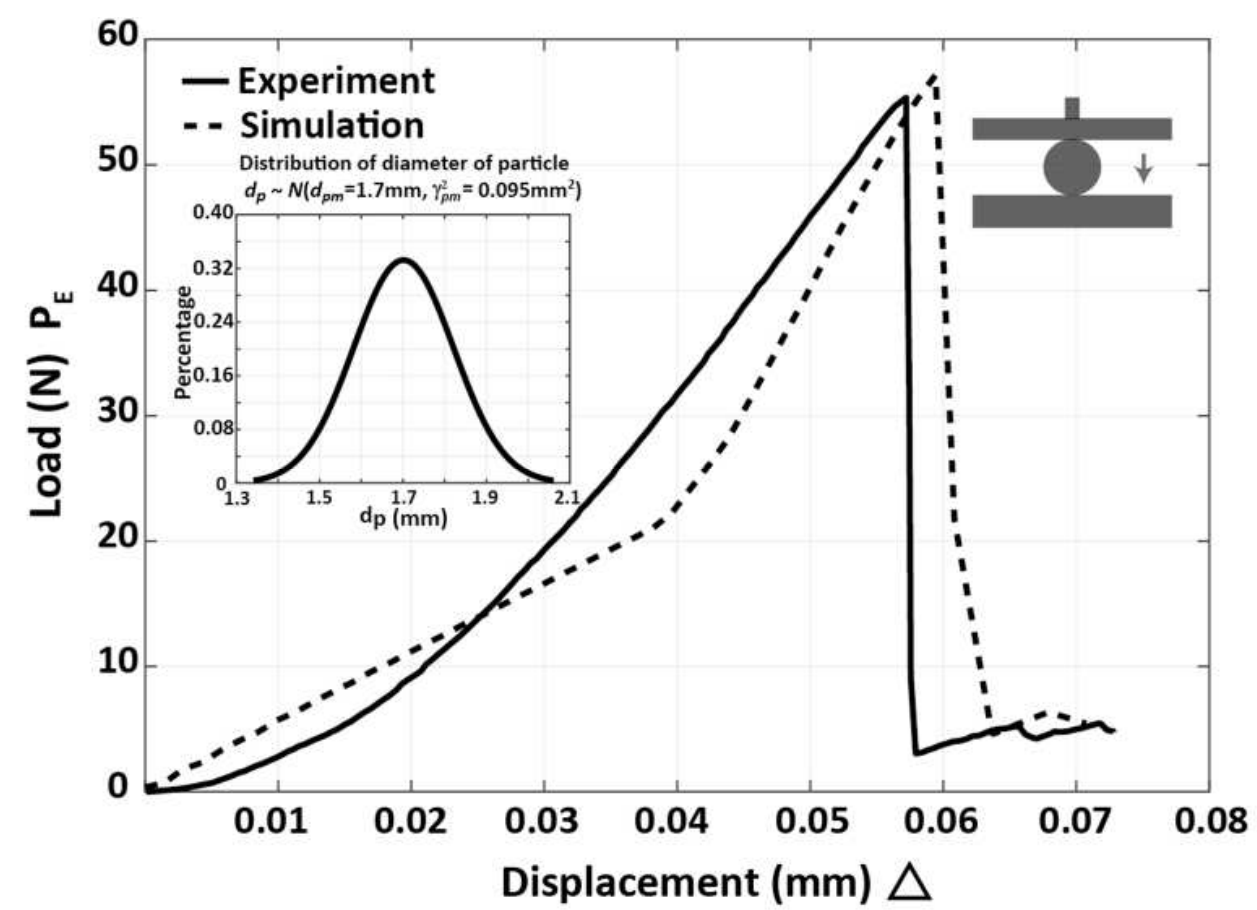

Fig 1. Compressive force as a function of the displacement of the movable crosshead of test machine obtained via single ceramic particle compression test and numerical simulation. The insert on the right schematically shows the compression test. The insert on the left shows the distribution of the diameters of the particles.

The LLDPE foams (including ceramic particle reinforced LLDPE foams and pure LLDPE foams) were manufactured using the method described as follows. A release agent was applied to the surface of a steel mould to ensure a smooth surface and provide ease of demoulding. Ceramic particles, LLDPE powder and azodicarbonamide blow agent were mixed together in a blender for 5 minutes. The mixture was then poured into a sealed steel mould and heated at $210{ }^{\circ} \mathrm{C}$ for 1 hour before cooling at room temperature for 24 hours.

The particle reinforced LLDPE foams are composed of three parts, i.e. particle reinforcements, LLDPE polymer (solid part) and hollow spherical cells (voids within a foam matrix). Throughout the paper, $F$ will be used to represent the volume fraction of polymer and $f$ to represent the 
volume fraction of particle reinforcement, i.e. $\mathrm{F}=V_{P} / V_{T}$ and $f=V_{f} / V_{T}$, where $V_{T}$ denotes the total volume of a foam sample, $V_{p}$ the volume of the LLDPE polymer inside the foam sample and $V_{f}$ the volume of particle reinforcement embedded in the foam sample. In addition, $M_{T}$ will be used to represent the total mass of a foam sample, $M_{p}$ the mass of polymer and $M_{f}$ the mass of particle reinforcement. Four types of pure foam samples were manufactured for testing, with $\mathrm{F}=0.214,0.267,0.321$ and 0.428 . For $\mathrm{F}<0.214$, there was insufficient LLPDE powder to form a foam sample; for $\mathrm{F}>0.428$, foams were too heavy to have practical applications. The particle reinforced foams manufactured for testing were $\mathrm{F}=0.214$ and 0.321 and $f=0.1,0.2$ and 0.3 . For $f>0.3$, a homogenous foam structure could not be formed.

In order to examine the internal porous structures of the LLDPE foams, two-dimensional (2D) images of sectioned foam samples were observed using a Nikon SMZ800 optical microscope. Figure 2 shows the microscopic images of pure foam samples at selected polymer volume fractions (F). The internal porous structures are composed of hollow spherical cells and polymer (separation) among the cells. The typical sketch of the internal porous structures is shown in Fig. 2 (b). Microscopic images in Fig. 2 suggest that the foams contains hollow spherical cells of different sizes. Cells sizes measured via 2D microscopic images may not be able to reflect the actual values (Chen et al., 2015). However, Fischer et al. (2009) demonstrated that the distributions of cells diameter measured from 2D microscopic images are similar to those measured by 3D reconstructed models. To understand the distribution of the diameter of the hollow cells within each foam sample, histograms of the distribution of cells based on 2D images are presented in Fig. 2. As polymer volume fraction $F$ increases, the cell diameter decreases, e.g. the measured cell diameter $D_{c}$ mainly varies from $0.464 \mathrm{~mm}$ to $3.824 \mathrm{~mm}$ with $\mathrm{F}=0.214$ and from $0.659 \mathrm{~mm}$ to $2.356 \mathrm{~mm}$ with $\mathrm{F}=0.267$. The distribution of the diameter of cells within each foam sample can be approximated as a normal distribution (Richardson et al., 2000; Marvi-Mashhadi et al., 2018; Verdolotti et al., 2015), $D_{c} \sim N\left(D_{c m}, \gamma_{c m}^{2}\right)$ with $D_{c m}$ representing the mean diameter of cells and $\gamma_{c m}^{2}$ the variance, as shown in Table 1 for selected volume fractions $\mathrm{F}$. In this table, 
separation $e$ is defined as the distance between any two adjacent hollow cells as shown in Fig. 2 (b).

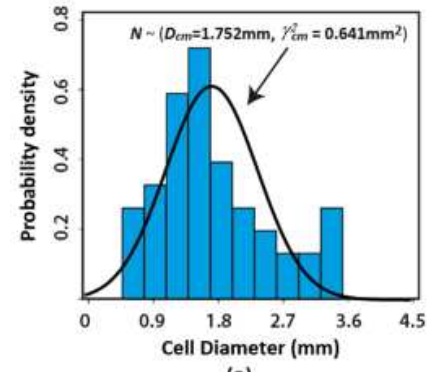

(a)

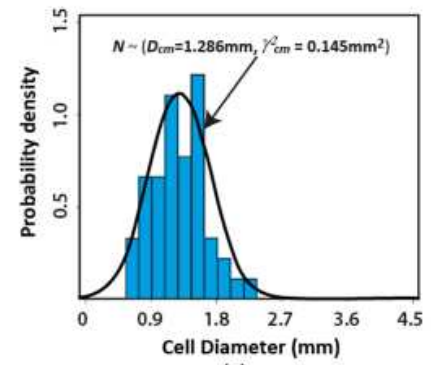

(c)

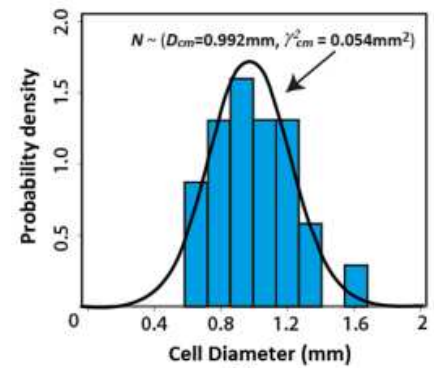

(e)

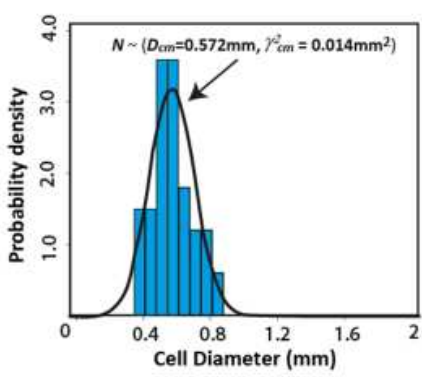

(g)

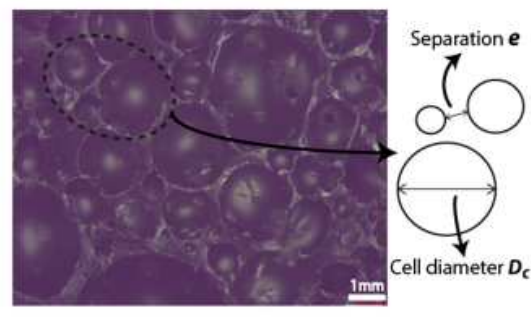

(b)

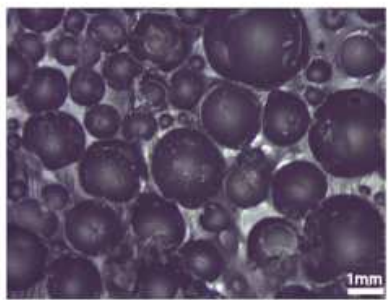

(d)

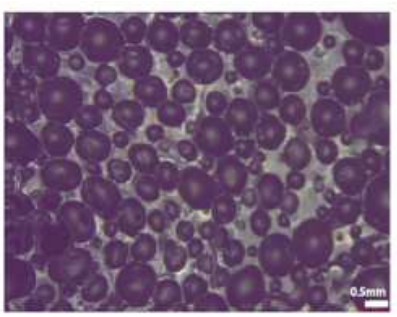

(f)

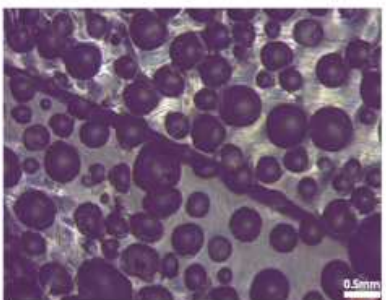

(h)

Fig 2. The distributions of the measured diameters of the hollow cells and microscopic images of the sectioned LLDPE pure foam samples with $F=0.214$ as shown in (a) and (b), $F=0.267$ as shown in (c) and (d), $F=0.321$ as shown in (e) and (f) and $F=0.428$ as shown in $(g)$ and $(h)$, respectively. (For interpretation of the color legend in this figure, the reader is referred to the web version of this article.) 
Figures $3(\mathrm{a})$ and (b) show the microscopic images of the sections of the particle reinforced LLDPE foams with $\mathrm{F}=0.214, f=0.1$ and $\mathrm{F}=0.214, f=0.2$, respectively. As shown in these images, the presence of the particle reinforcement within the polymer foam does not significantly alter the diameters of the hollow spherical cells inside the polymer foams. As the ceramic particles are brittle, some particles were damaged while sectioning the specimen as shown in Fig. 3(b). Figures 3 (c) and (d) show the X-ray computed tomography (CT) images showing the internal structure of particle reinforced foams with $\mathrm{F}=0.214, f=0.1$ and $\mathrm{F}=0.214, f=0.2$, respectively. It can be seen that the particle reinforcements were reasonably distributed within the foam matrix.

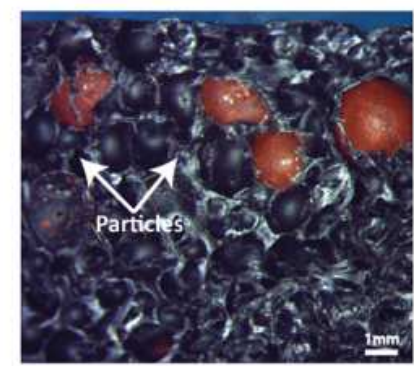

(a) $\mathrm{F}=0.214, f=0.1$

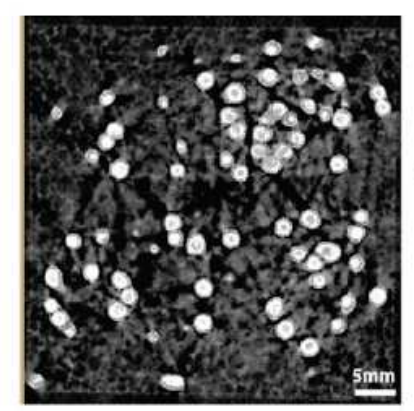

(c) $\mathrm{F}=0.214, f=0.1$

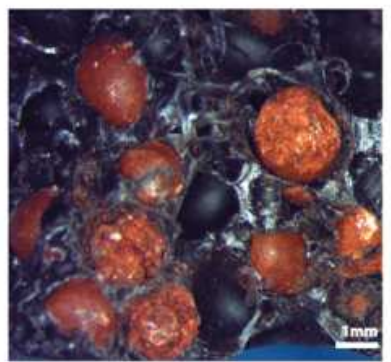

(b) $\mathrm{F}=0.214, f=0.2$

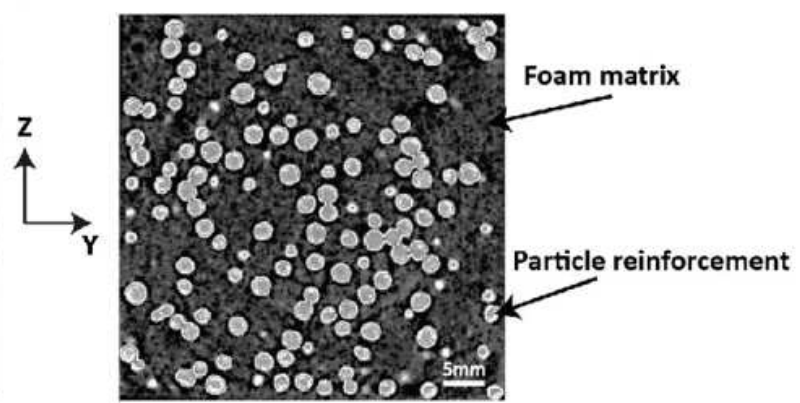

(d) $\mathrm{F}=0.214, f=0.2$

Fig 3. Microscopic images and X-ray $C T$ images of the particle reinforced foam samples with $\mathrm{F}=0.214, f=0.1$ as shown in (a) and (c) and $\mathrm{F}=0.214, f=0.2$ as shown in (b) and (d), respectively. (For interpretation of the color legend in this figure, the reader is referred to the web version of this article.)

\section{Experimental protocol}

Quasi-static compressive tests implemented on LLDPE foams were carried out in a screw-driven Zwick/Roell Universal testing machine at room temperature following the procedure defined by 
ASTM 1621-04a. Cubic specimens with an edge length of $I=50 \mathrm{~mm}$ were used for both pure foams and particle reinforced foams. Tests were conducted at a crosshead speed of $5 \mathrm{~mm} / \mathrm{min}$ with three samples for each type of foams. The measured compressive force $P_{F}$ and the vertical displacement $\Delta l$ of the crosshead were recorded by the testing machine. The nominal compressive strain and stress of the foams were calculated as $\varepsilon=\left|I-I_{0}\right| /\left|I_{0}\right|$ and $\sigma=P_{F} / A$, respectively, where $I_{0}$ and $I$ are the original height and final height of the foams, respectively; $A$ is the original cross-sectional area of the foams, $A=I_{0}^{2}$.

\section{Finite element simulations}

Finite element (FE) simulations of the quasi-static compressive response of LLDPE foams have been conducted to

1. verify the proposed FE calculations in predicting the quasi-static compressive response of LLDPE foams;

2. compare with experimental measurements for interpretation purposes;

3. investigate the reinforcing mechanism caused by the interactions between ceramic particles and hollow spherical cells during compression; and

4. analyse the size effects of particles and hollow cells.

\subsection{Generation of the geometry of foam structures}

The hollow spherical cells and ceramic particles within a foam sample were modelled explicitly as spheres that were randomly inserted into the cubic domain of the sample. The diameter of the cells $D_{c}$ and the diameter of particles $d_{p}$ are assumed to follow a normal distribution $D_{c} \sim N\left(D_{c m}, \gamma_{c m}^{2}\right)$ and $d_{p} \sim N\left(d_{p m}, \gamma_{p m}^{2}\right)$, respectively. Here, $D_{c m}$ denotes the mean diameter of inserted hollow cells; $\gamma_{c m}^{2}$ the variance of inserted hollow cells; $d_{p m}$ the mean diameter of inserted particles; and $\gamma_{p m}^{2}$ the variance of inserted particles. The following algorithms are applied to place all the spherical insertions, regardless of whether represent particles or hollow cells. 
The location of a sphere within the cubic domain satisfies two conditions.

- coordinates $(x, y, z)$ of the centre of the sphere obey the following relation

$$
(x, y, z) \in\{(x, y, z) \mid r \leq x \leq I-r, r \leq y \leq I-r, r \leq z \leq I-r\}
$$

where $r$ denotes the radius of a sphere, i.e. $r=D_{c} / 2$ for cells or $r=d_{p} / 2$ for particles.

- Spheres do not overlap with each other, i.e.

$$
\sqrt{\left(x_{i}-x_{j}\right)^{2}+\left(y_{i}-y_{j}\right)^{2}+\left(z_{i}-z_{j}\right)^{2}} \geq r_{i}+r_{j}+\tilde{e}, \forall i, j \in n, i \neq j
$$

where $n$ is the total number of spheres, and $\tilde{e}$ the mean separation defined as the mean distance between any two adjacent hollow cells. The generation of the geometry of a particle reinforced foam structure was achieved using a Python script within the environment of the commercially available FE software ABAQUS ${ }^{\circledR}$ using a 3-step procedure, as shown schematically in Figure 4. After the creation of the cubic domain of a foam sample, solid spheres which have the same diameter as the hollow cells and particles were inserted randomly into the cubic domain without overlap (Step 1). A Boolean operation was then conducted to remove the solid spheres from the cubic domain (Step 2). Then, solid spheres corresponding to the required solid particles were created again (Step 3). Figure 4 also shows the coordinate system used in the simulations. Throughout the paper, the $Z$-axis is parallel to the compression direction, and the $X$-axis and $y$ axis are perpendicular to the compressive direction. 


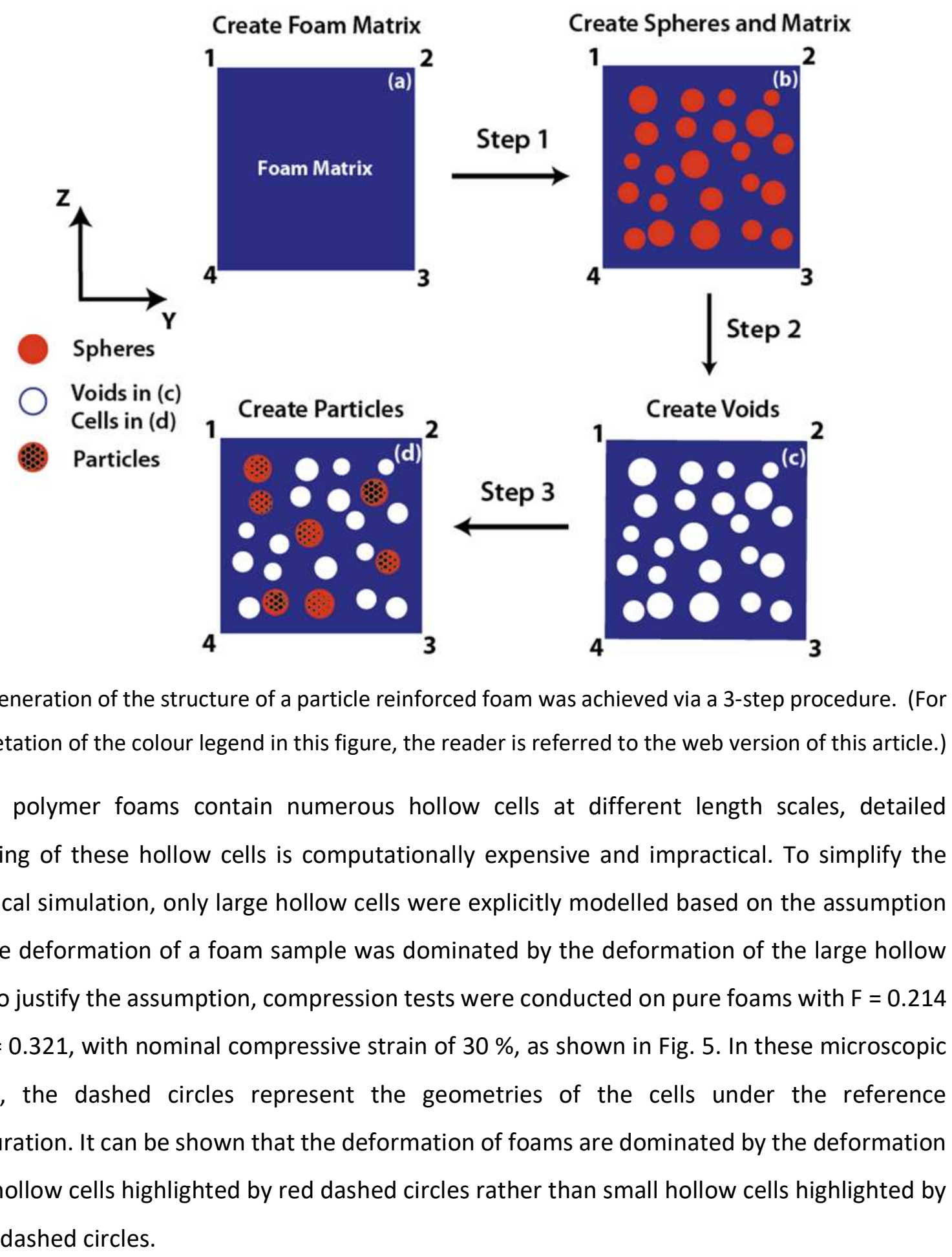



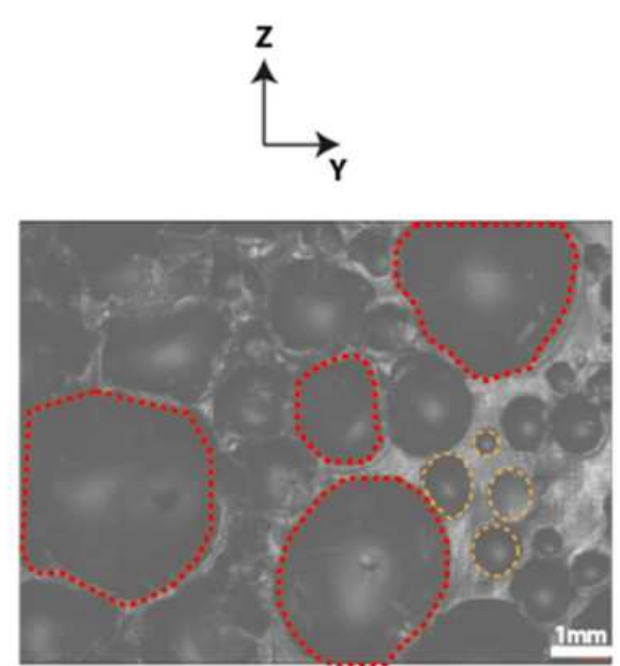

(a)
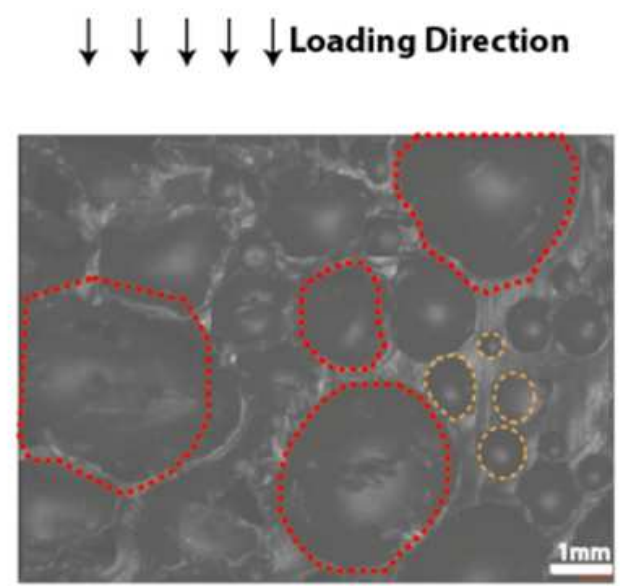

(c) $\because ;$ : Large cells

: Small cells

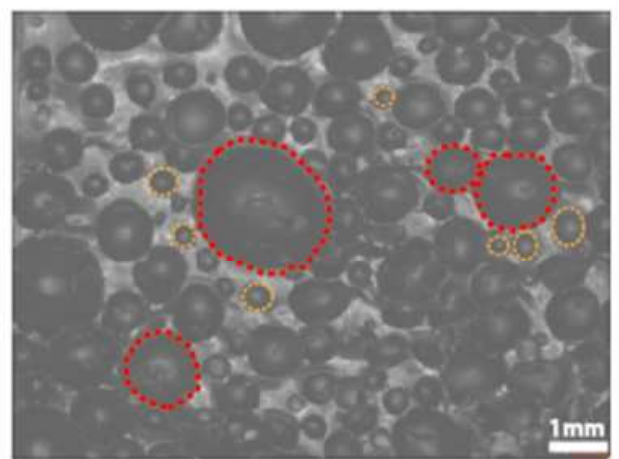

(b)
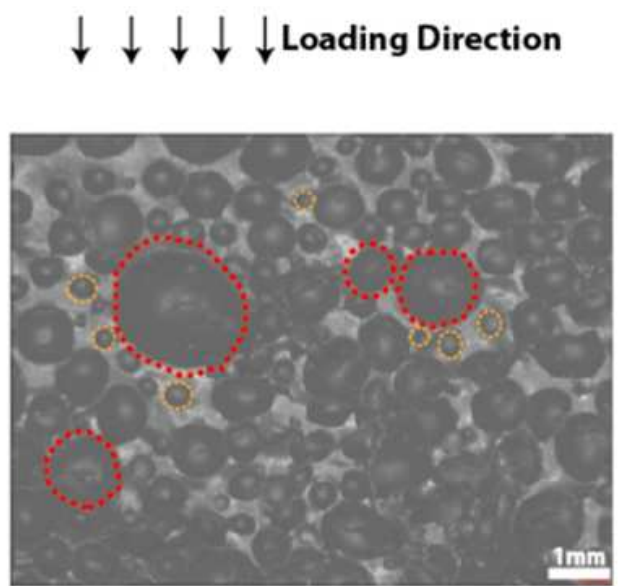

(d)

Fig 5. Microscopic images of the sectioned LLDPE pure foam samples before compression for (a) $\mathrm{F}=0.214$ and (b) $\mathrm{F}=0.321$; and after compression with permanent compressive strain $\varepsilon=0.3$ for (c) $F=0.214$ and (d) $F=0.321$. (For interpretation of the color legend in this figure, the reader is referred to the web version of this article.)

Based on the assumption, the modelling strategy is that only large hollow cells were inserted into a parent foam matrix of higher density to create the numerical model of a lower density foam to avoid heavy computation. The constituent model presented in Section 4.3.1 was used to model the behaviour of the fine-pored parent foam matrix. The modelling strategy can be schematically shown in Fig. 6 for the creation of the FE model for the pure foam samples with $F=0.321$. The 
pure foam with $F=0.321$ has the measured cells diameter range of $0.306 \mathrm{~mm}$ to $1.772 \mathrm{~mm}$, and follows $D_{c} \sim N\left(D_{c m}=0.992 \mathrm{~mm}, \gamma_{c m}^{2}=0.054 \mathrm{~mm}^{2}\right)$; the pure foam with $F=0.428$ has the measured cells diameter range of $0.287 \mathrm{~mm}$ to $0.931 \mathrm{~mm}$, and follows $D_{c} \sim N\left(D_{c m}=0.572 \mathrm{~mm}, \gamma_{c m}^{2}=0.014 \mathrm{~mm}^{2}\right)$. Hence, the FE model with $\mathrm{F}=0.321$ was created via inserting hollow cells with diameter range of $0.931 \mathrm{~mm}$ to $1.772 \mathrm{~mm}$ and following $D_{c} \sim N\left(D_{c m}=1.56 \mathrm{~mm}, \gamma_{c m}^{2}=0.01 \mathrm{~mm}^{2}\right)$ to the foam matrix $(F=0.428)$. Similarly, the FE model of pure foam with $F=0.214$ was created based on the foam matrix with $F=0.267$ with the same modelling strategy, see Appendix A.

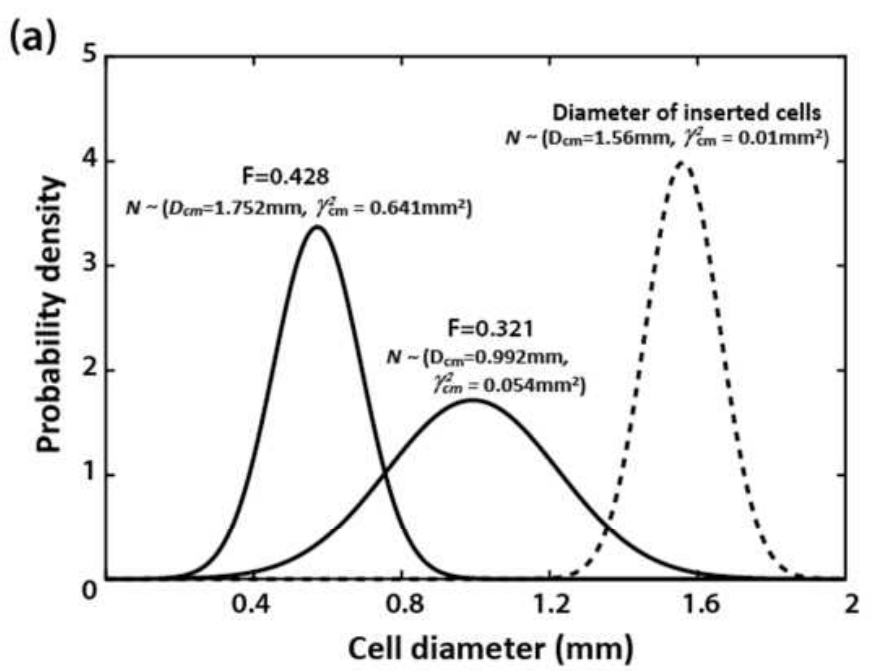

(b)

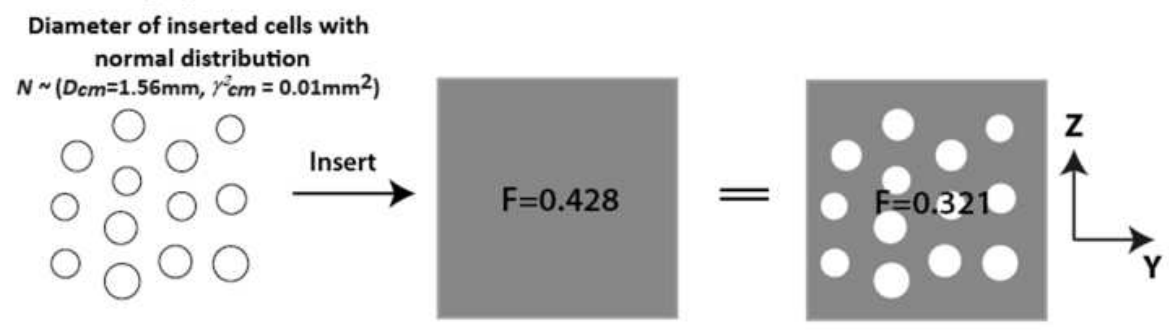

Fig 6. Creation of the numerical models for pure foams with $F=0.321$. (a) The distributions of cells diameter for the pure foams with $F=0.321$ and $F=0.428$ as well as the inserted cells. (b) The hollow cells which follow $D_{c} \sim N\left(D_{c m}=1.56 \mathrm{~mm}, \gamma_{c m}^{2}=0.01 \mathrm{~mm}^{2}\right)$ were inserted to the foam matrix with $\mathrm{F}=0.428$ to create the FE model of the pure foam with $\mathrm{F}=0.321$. (For interpretation of the color legend in this figure, the reader is referred to the web version of this article.) 


\subsection{The finite element model}

The explicit version of the commercially available finite-element (FE) package ABAQUS was used for the FE calculations. The foam matrix was modelled by a four-node tetrahedral element (C3D4 in ABAQUS notation) which enables automated meshing of complex geometries. A numerical study confirmed that a maximum element edge length of $1 / 20$ the edge length of foam domain was required to achieve converged results. The foam domain consisted of over 250,000 elements. The particles were modelled by eight-nodded brick element with reduced integration element (C3D8R). Numerical tests suggested that a maximum element side length of $1 / 26$ of the diameter of a particle was required to achieve converged results and capture the damage occurring in the particle. The number of elements for each particle was in the range of 830 to 1290 depending on the diameter. The FE model for a cubic foam sample is shown in Fig. 7.
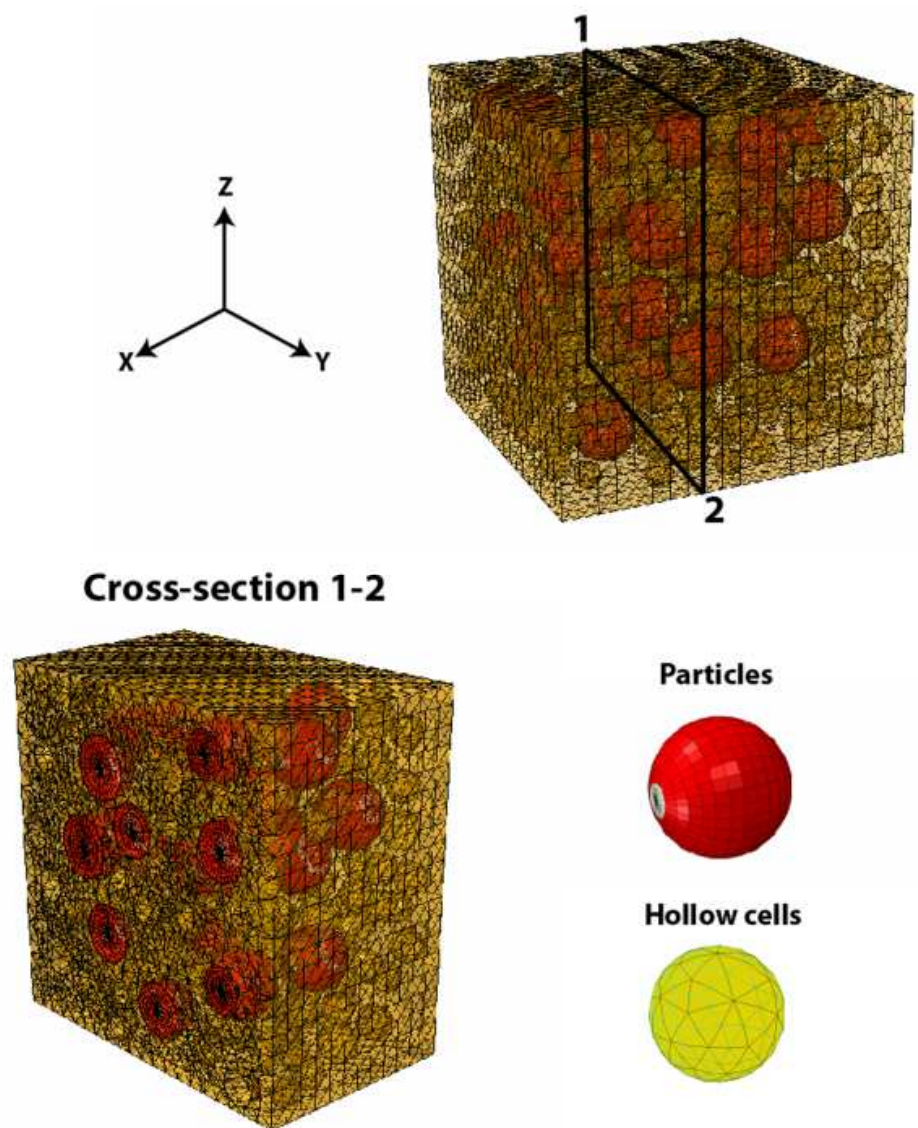

Fig 7. Finite element model for a cubic particle reinforced foam sample. (For interpretation of the color legend in this figure, the reader is referred to the web version of this article.) 
To simulate the compression test, the FE model of a cubic foam sample was sandwiched between two rigid plates (discretised with the 4-node rigid elements, R3D4): one of the rigid plates was stationary, and the other rigid plate was movable along the $z$-axis direction. The movable rigid plate imposed pressure on the sample at a constant velocity. To ensure a quasi-static simulation, the velocity was controlled to keep the kinetic energy under $5 \%$ of the total energy in the system. A penalty contact approach was employed to simulate the interaction between all surfaces with a friction coefficient 0.2 . The cohesive effect between the foam matrix and particle reinforcement was ignored owing to the significant difference in material stiffness of the two materials. Numerical trial tests suggested that the simulation results were not sensitive to the value of the friction coefficient employed in the calculations.

\subsection{Material constitutive models}

\subsubsection{The LLDPE foam matrix}

The isotropic crushable foam model proposed by Deshpande and Fleck (2000) was employed to model the mechanical behaviour of the LLDPE foams. The isotropic yield function $\Phi$ of the constitutive model is described by

$$
\Phi=\hat{\sigma}-Y=0
$$

where $Y$ is the uniaxial yield stress and $\hat{\sigma}$ denotes the equivalent stress which is a function of von Mises stress $\sigma_{e}$ and mean stress $\sigma_{m}$ (Deshpande and Fleck, 2000), given as

$$
\hat{\sigma}^{2}=\frac{\sigma_{e}^{2}+\theta^{2} \sigma_{m}^{2}}{1+\theta^{2} / 9}
$$

where $\theta$ defines the shape factor of the yield surface and can be calculated from the plastic Poisson's ratio $v_{p}$

$$
\theta^{2}=\frac{9\left(1-2 v_{p}\right)}{2\left(1+v_{p}\right)}
$$


In the crushable foam model, the yield stress ratio $k$ is defined as the ratio of initial yield stress $\sigma_{0}$ in the uniaxial compression to the initial yield stress $p_{0}$ in hydrostatic compression. The nonassociated flow rule for the isotropic model also gives $k$ as

$$
k=\sqrt{3\left(1-v_{p}\right)}
$$

In the FE simulation, the LLDPE foam was assumed to have a plastic Poisson's ratio $v_{p}=0$. Hence, shape factor $\theta$ and yield stress ratio $k$ were calculated as 2.12 and 1.73 , respectively. The stressstrain curves obtained from uniaxial quasi-static compressive tests were employed to calibrate the post yielding behaviour of LLDPE foams in the FE simulations.

\subsubsection{The Ceramic particles}

The Rankine criterion (Dahmani and Mohand, 2011) was employed to detect the crack initiation within ceramic particles, i.e. a crack forms when the principal stress reaches the tensile strength $\sigma_{t m}$. The fracture energy based crack evolution criterion defined by Hillerborg et al. (1976) was employed to simulate the post-failure behaviour of the ceramic particles. Once cracks are initiated, the stress at a material point decreases linearly with the increasing of crack opening displacement $u_{t}$, see Figure $8(a)$. Energy $W_{c}$ is required to open a unit area of a crack and can be calculated as

$$
W_{c}=\int_{0}^{u_{t m}} \sigma_{t} d u_{t}
$$

where $u_{t m}$ denotes the crack opening displacement when the stress $\sigma_{t}$ decreases to zero. After crack initiation, the shear modulus of a material will decrease, as given below

$$
G_{s}=\psi G
$$

where $\psi$ denotes a shear retention factor that is a function of crack opening strain $\varepsilon_{s}$; $G$ the shear modulus for the uncracked parent material; $G_{s}$ the post-failure shear modulus. In the numerical simulations, $\psi$ was assumed to evolve with $\varepsilon_{s}$ based on a bilinear model, as shown in 
Fig. 8(b), with $\left(\psi_{m}, \varepsilon_{m}\right)$ representing the mid-point and $\varepsilon_{s m}$ the crack opening strain when $\psi=0$. Bilinear softening model were employed by Roesler et al. (2006) to simulate the load versus crack opening of the notched concrete specimens under three point bending, which was shown to be in good agreement with experimental measurements. In the FE simulation of this study, the element deletion technique was employed to remove elements from meshes when the deformation of the element reached the crack opening displacement $u_{t m}$ to avoid excessive element distortion. The parameters used in this study are shown in Table 2.
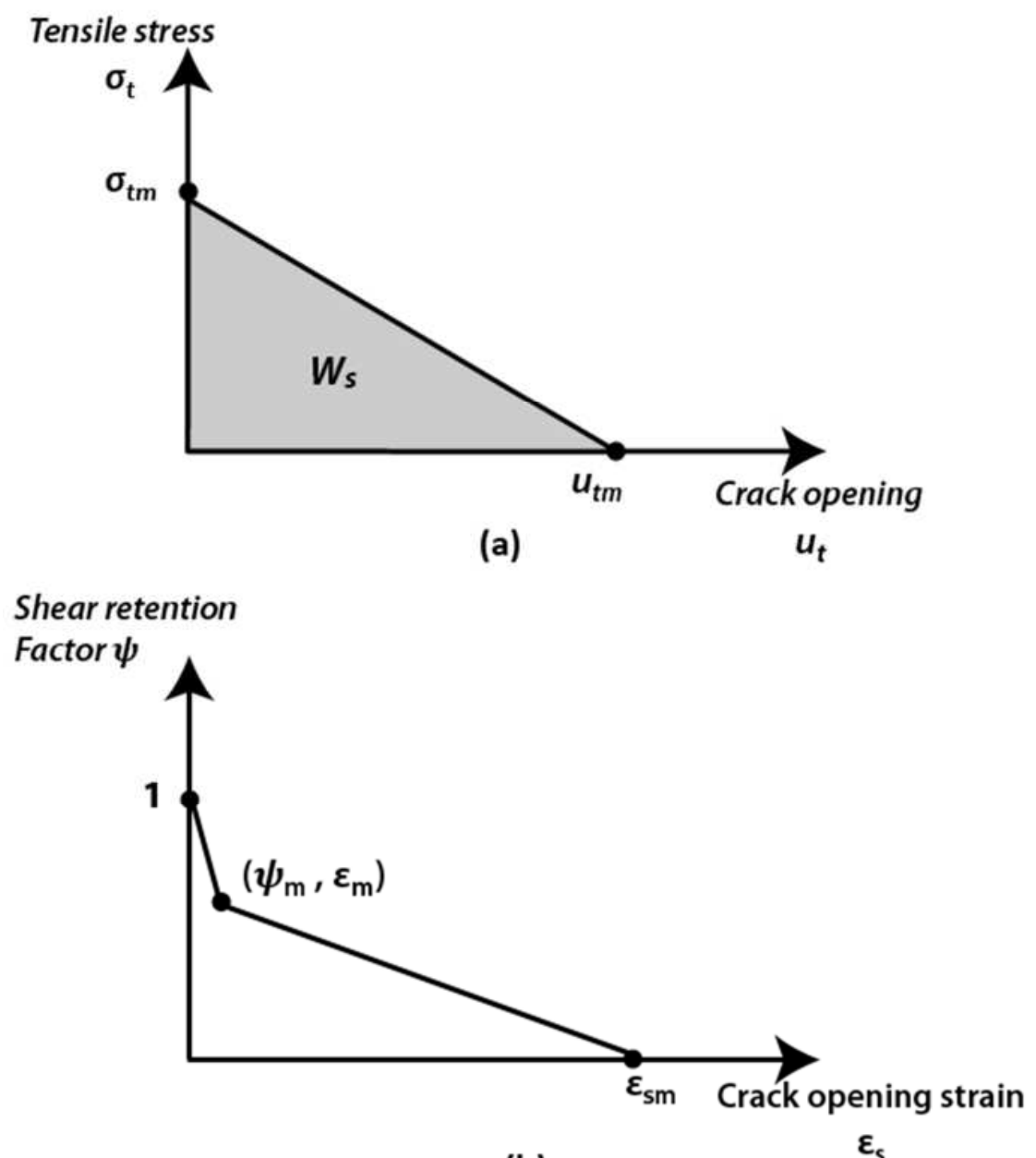

(b)

Fig 8. (a) The relation between tensile stress and crack-opening displacement after a crack is initiated; (b) The bilinear softening model defines the relation between shear retention factor and crack opening strain. 


\section{Results and discussions}

\subsection{Experimental measurements}

The measured quasi-static compressive response of the foam samples are plotted in Fig. 9 (a) for pure foams and Figs. 10 (b) and (c) for particle reinforced foams. Under uniaxial compression, the stress-strain curves of the LLDPE foams consist of three regions, i.e. elastic region, plateau region and densification region. The yield strength and elastic moduli of LLDPE foams observed from quasi-static compression are summarised in Table 3 . In the following description, $E_{f}, \sigma_{f}$ and $\rho_{f}$ represent the elastic modulus, yield strength and density of a pure foam; $E_{c}, \sigma_{c}$ and $\rho_{c}$ represent the elastic modulus, yield strength and density of a particle reinforced foam.

\section{The pure LLDPE foams}

As illustrated in Table. 3, the yield strength $\sigma_{f}$ and elastic modulus $E_{f}$ of the pure LLDPE foams increase with the increase of foam volume fraction F . Gibson and Ashby (1997) and Ashby (1993) have suggested $\sigma_{f} / E_{s} \propto \tilde{\rho}^{2}$ for closed-cell foams, see Eq. (A.2) in Appendix B, where $\tilde{\rho}$ is the relative density of the foams, $\tilde{\rho}=\rho_{f} / \rho_{s}$ ( $\tilde{\rho}$ equates $F$ for pure foams). Figure 9 (b) shows that the experimental measurements are in good agreements with the predictions provided by Eq. (A.2). Figure 9 (c) shows the experimental measurements of elastic modulus versus volume fraction of polymer $F$. It also demonstrates that, with curve fitting, $E_{f}$ is quadratic with $F$, see Appendix C, Eq. (B.2). 

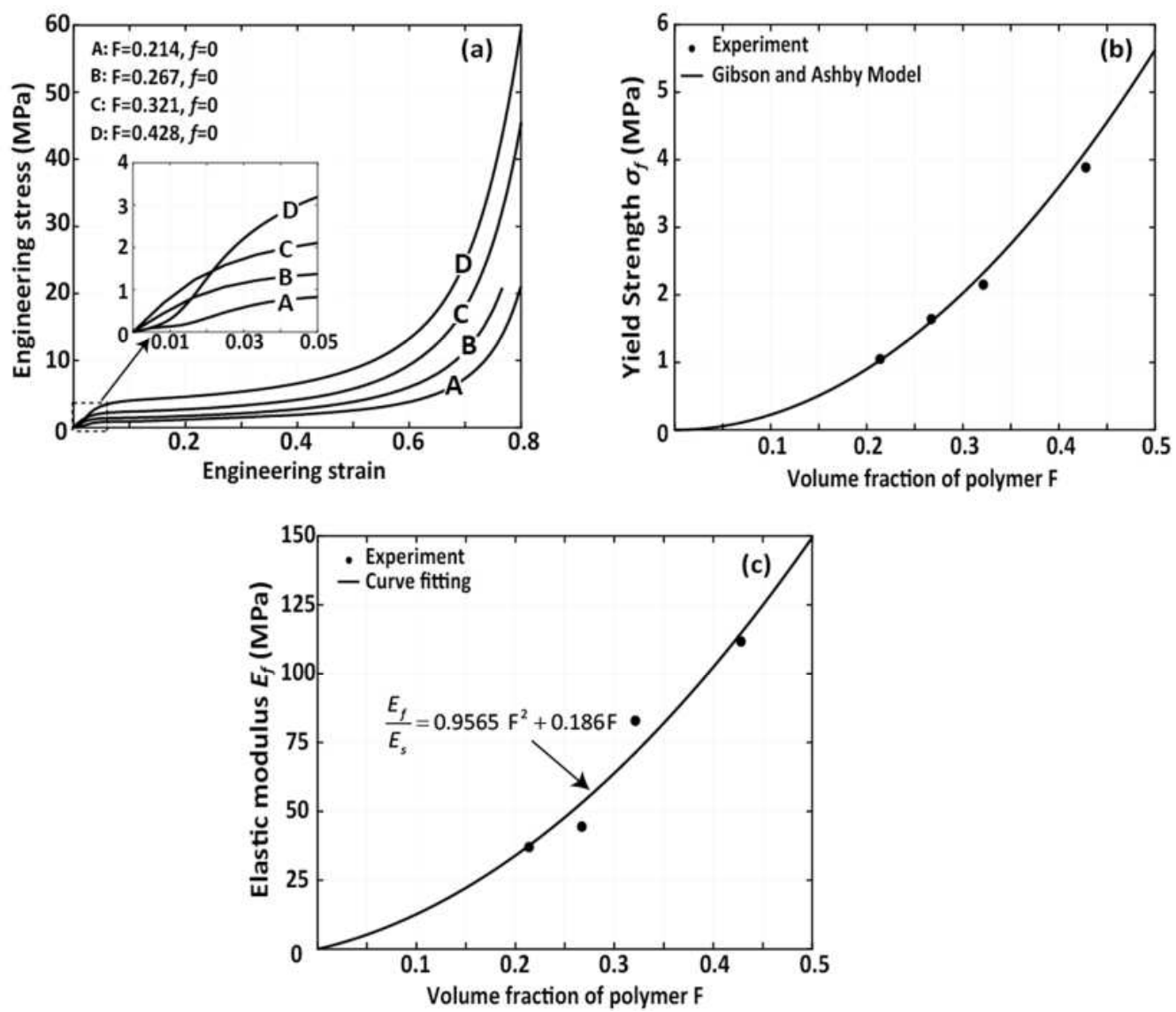

Fig 9. (a) The uniaxial stress-strain relation of pure LLDPE foams at selected polymer volume fractionF. (b) The relation of yield strength $\sigma_{f}$ and volume fraction $\mathrm{F}$ for pure LLDPE foams is compared between experimental measurements and theoretical predictions obtained from Gibson and Ashby model (1997). (c) Elastic modulus $E_{f}$ of pure foams as a function of volume fraction $\mathrm{F}$. Here, $E_{s}$ is the elastic modulus of the LLDPE powder.

\section{The Particle reinforced foams}

The effects of the particle reinforcements are shown in all three regions from Figs. 10 (a) and (b): (1) enhancement in elastic modulus and yield strength in the elastic region; (2) apparent hardening in the plateau region; and (3) higher stresses in the densification region. The measured yield strength $\sigma_{c}$ and elastic modulus $E_{c}$ increase with the increase of the volume fraction of particle reinforcements $f$, as illustrated in Table. 3. Figure 10 (c) shows the yield strength $\sigma_{c}$ as a function of volume fraction $f$. The yield strength predicted by Eq. (A.4) in Appendix B, which 
suggests that $\sigma_{c} \propto\left(E_{s}, \tilde{\rho}, \sigma_{p}, f\right)$, is used to compare with that obtained from experimental measurements. The agreements between the predictions and the experimental measurements are good except for the cases with higher particle reinforcement volume fraction, i.e. $f>0.2$, as shown in Fig. 10 (c). Kernel (1956), Isai and Cohen (1967) and Halpin and Tsai (1969) have suggested, for particle reinforced composites, $E_{c} \propto\left(E_{f}, E_{p}, f\right)$, see Eqs. (B3), (B6) and (B8) in Appendix C. The comparisons between the predictions based on these models and experimental measurements for elastic modulus $E_{c}$ show good agreements, as shown in Fig. 10 (d).
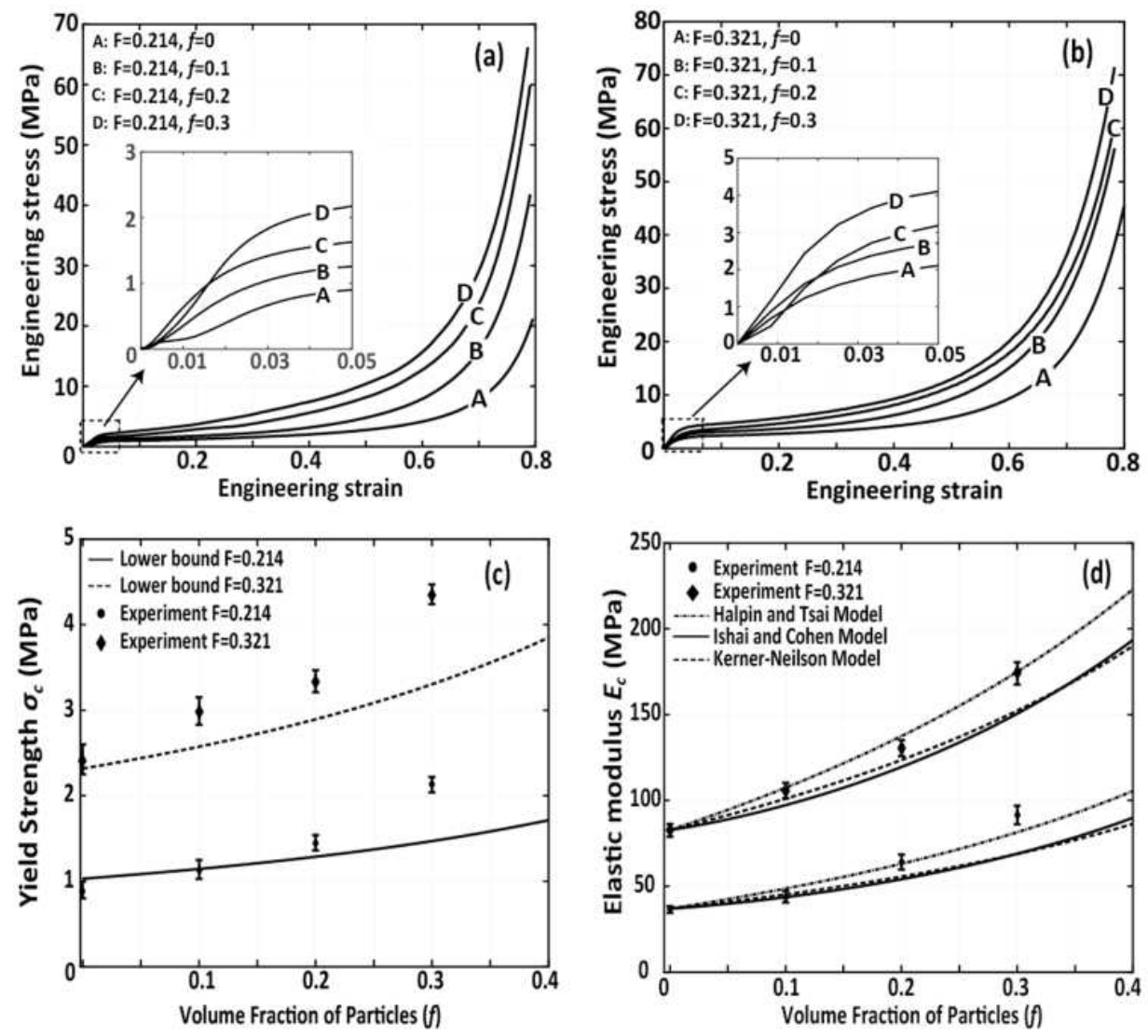

Fig 10. The uniaxial stress-strain relations of ceramic particle reinforced LLDPE foams with F=0.214 and $\mathrm{F}=0.321$ under quasi-static compression are shown in (a) and (b), respectively. Yield strength $\sigma_{c}$ and elastic modulus $E_{c}$ as a function of volume fraction $f$ are compared between experimental measurements and theoretical predictions obtained from Ruess lower bound (see Appendix B) and analytical models (see Appendix C) are shown in (c) and (d), respectively. 


\section{Energy absorption}

Materials with good energy absorption capability have the potential application in automotive industrial to absorb impact energy during crashing (Mondal et al., 2009). For foam materials, deformations of the hollow cells within the foams absorb most energy before densification (Gibson and Ashby, 1997; Liu and Gong, 2006; Ashby, 2006; Evans et al., 2010). With the presence of particle reinforcements, hollow cells could be stabilised by particle reinforcements to achieve higher macroscopic stiffness and strength. Numerical simulations have suggested that densification starts to develop at $\varepsilon=0.5$ (Section 5.2.2), i.e. the top and bottom sides of the large hollow cells are nearly in contact with each other. Figure 11 shows a typical stress-strain curve of LLDPE foams which illustrates elastic region, yield point (Point A), plateau region, onset of densification (Point B) and densification region. The absorbed energy per unit volume for $\varepsilon \leq 0.5$, i.e. $W_{1}=\int_{0}^{0.5} \sigma d \varepsilon$, is calculated to evaluate the effect of the particle stabilization, as schematically shown in Fig. 11. It has been observed that the foam samples are fully densified at $\varepsilon=0.8$ (see Figs 9 and 10), i.e. the top and bottom sides of the hollow cells are fully in contact with each other and particle reinforcement partially crushed. The absorbed energy per unit volume when $0.5 \leq \varepsilon \leq 0.8$, i.e. $W_{2}=\int_{0.5}^{0.8} \sigma d \varepsilon$, is calculated to evaluate the effect of particle crushing (Fig. 11). 


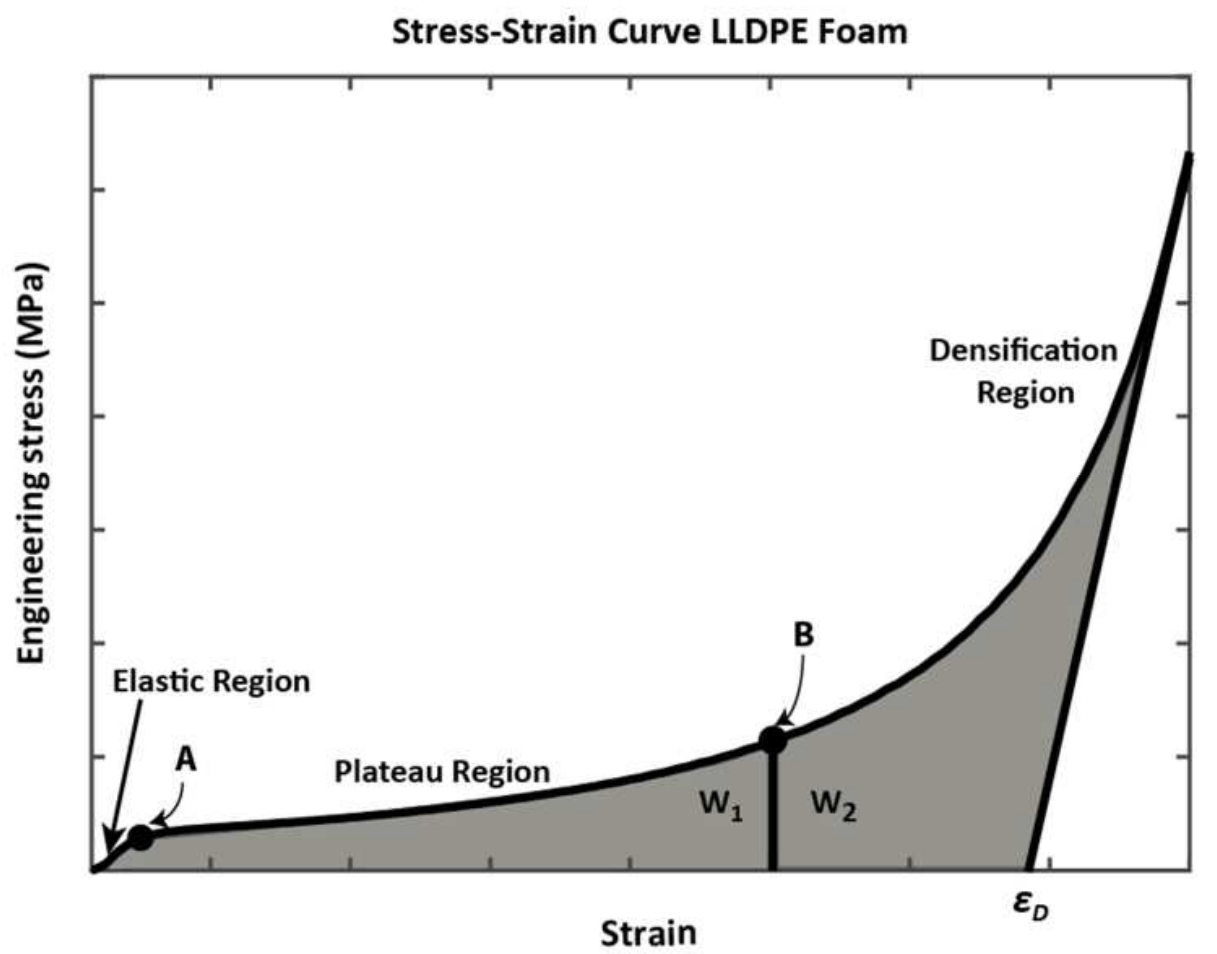

Fig 11. The typical stress-strain curve of LLDPE foams under compression contains elastic region, yield point (Point A), plateau region, onset of densification (Point $B$ ) and densification region.

To understand the capacity of energy absorption affected by particle reinforcements, the absorbed energy per unit volume of particle reinforced foams $W_{1-R}(\varepsilon \leq 0.5)$ and $W_{2-R}$ $(0.5 \leq \varepsilon \leq 0.8)$ are normalised by the absorbed energy per unit volume of the pure foams $W_{1-p}$ $(\varepsilon \leq 0.5)$ and $W_{2-p}(0.5 \leq \varepsilon \leq 0.8)$, respectively. The normalised energy absorptions of $\widehat{W}_{1}=W_{1-R} / W_{1-p}$ and $\widehat{W}_{2}=W_{2-R} / W_{2-p}$ as the functions of volume fraction of particle reinforcement $f$ for two selected polymer volume fractions, i.e. $\mathrm{F}=0.214$ and $\mathrm{F}=0.321$ are shown in Figures 12 (a) and (b), respectively. From Fig. 12, the normalised energy absorption $\widehat{W}_{1}$ and $\widehat{W}_{2}$ increase with the increase of particle volume fraction $f$, which suggests the particle reinforcement can enhance the capacity of energy absorption before and at the start of the densification, respectively. The enhancement of energy absorption capacity by particle reinforcement for lower polymer volume fraction, e.g. $\mathrm{F}=0.214$ is more effective than that for higher polymer volume fraction, e.g. $F=0.321$. 

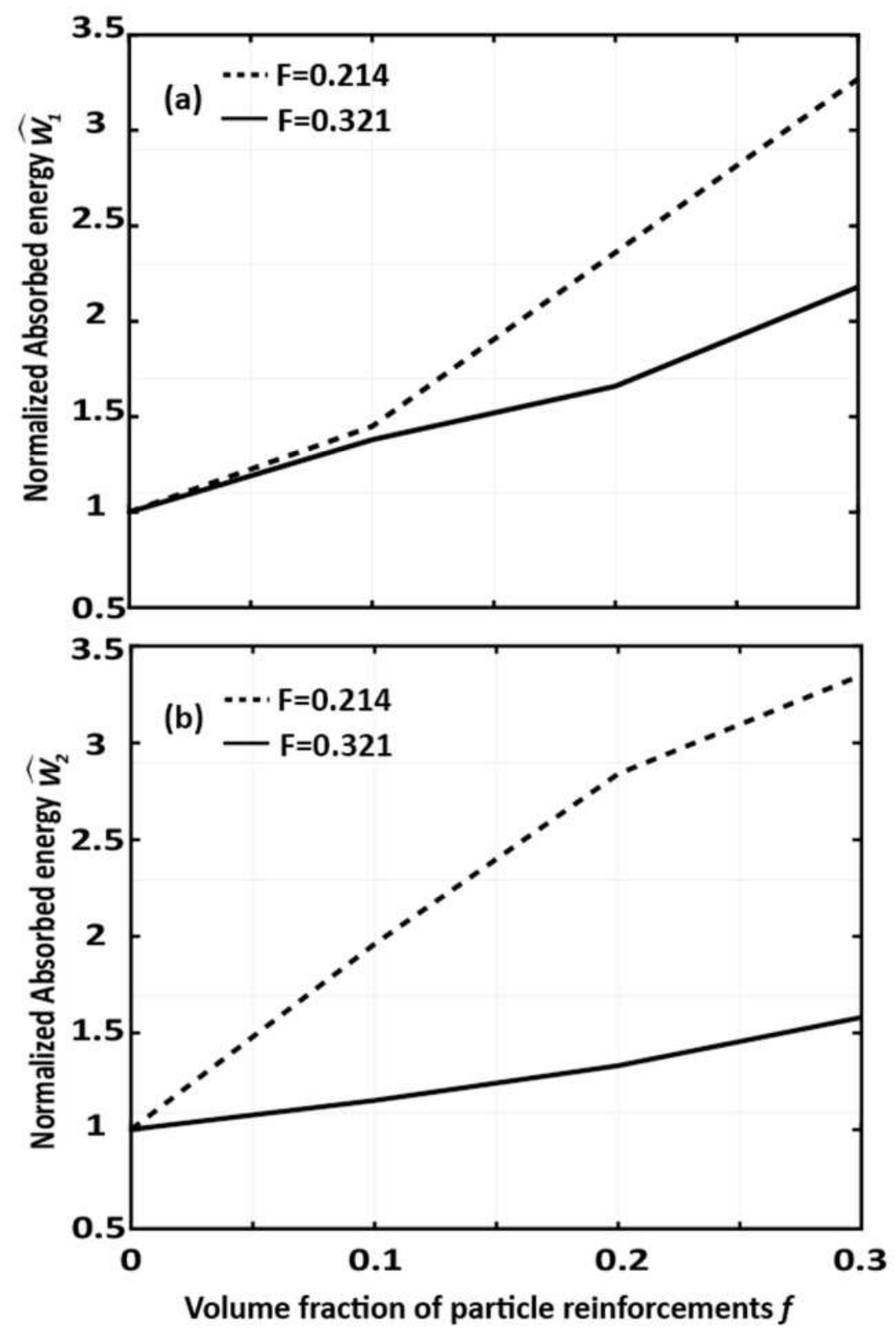

Fig 12. The absorbed energy per unit volume of particle reinforced foams normalised by the absorbed energy per unit volume of the pure foams, $\widehat{W}_{1}\left(\varepsilon \leq 0.5\right.$, (a)) and $\widehat{W}_{2}(0.5 \leq \varepsilon \leq 0.8$, (b)), as functions of volume fraction of particle reinforcement $f$ for two selected polymer volume fractions, i.e. $\mathrm{F}=0.214$ and $\mathrm{F}=0.321$.

To compare the energy absorption efficiency of pure foams and particle reinforced foams before the onset of densification (stabilisation effect), the absorbed energy per unit volume $W_{1-p}$ and 
$W_{1-R}$ are normalised by the elastic modulus of $E_{s}$ (LLDPE powder) for pure foams and $E_{c s}$ (mixtures of LLDPE powder and ceramic particles) for particle reinforced foams, respectively, where $E_{c s}=E_{s} E_{p} /\left(f E_{s}+(1-f) E_{p}\right)$ obtained from inverse rule of mixtures. The normalized absorbed energy per unit volume $U=W_{1-P} / E_{s}$ for pure foam or $U=W_{1-R} / E_{c s}$ for particle reinforced foam are plotted against normalized yield strength $\bar{\sigma}=\sigma_{f} / E_{s}$ for pure foam or $\bar{\sigma}=\sigma_{c} / E_{c s}$ for particle reinforced foam, respectively, as shown in Fig. 13. Figure 13 can be used to select the foams with required energy absorption capacity up to the maximum permitted stress $(\bar{\sigma})$ in the application of packaging materials (Ashby et al., 1985; Gibson and Ashby, 1997). Two kinds of closed-cell foams are shown in the figure: elastomeric foams investigated by Gibson and Ashby (1997) and LLDPE foams studied in this research. Compared to pure LLDPE foams, elastomeric foams exhibit higher energy absorption capacity at a given maximum permitted stress $\bar{\sigma}$. However, particle reinforced LLDPE foams exhibit the highest energy absorption efficiency among them. The reinforcing effect is again more significant for lower polymer volume fraction F. Figure 13 also suggests that the energy absorption capability increases with increase of particle volume fraction $f$ at both $\mathrm{F}=0.214$ and 0.321 , respectively. 


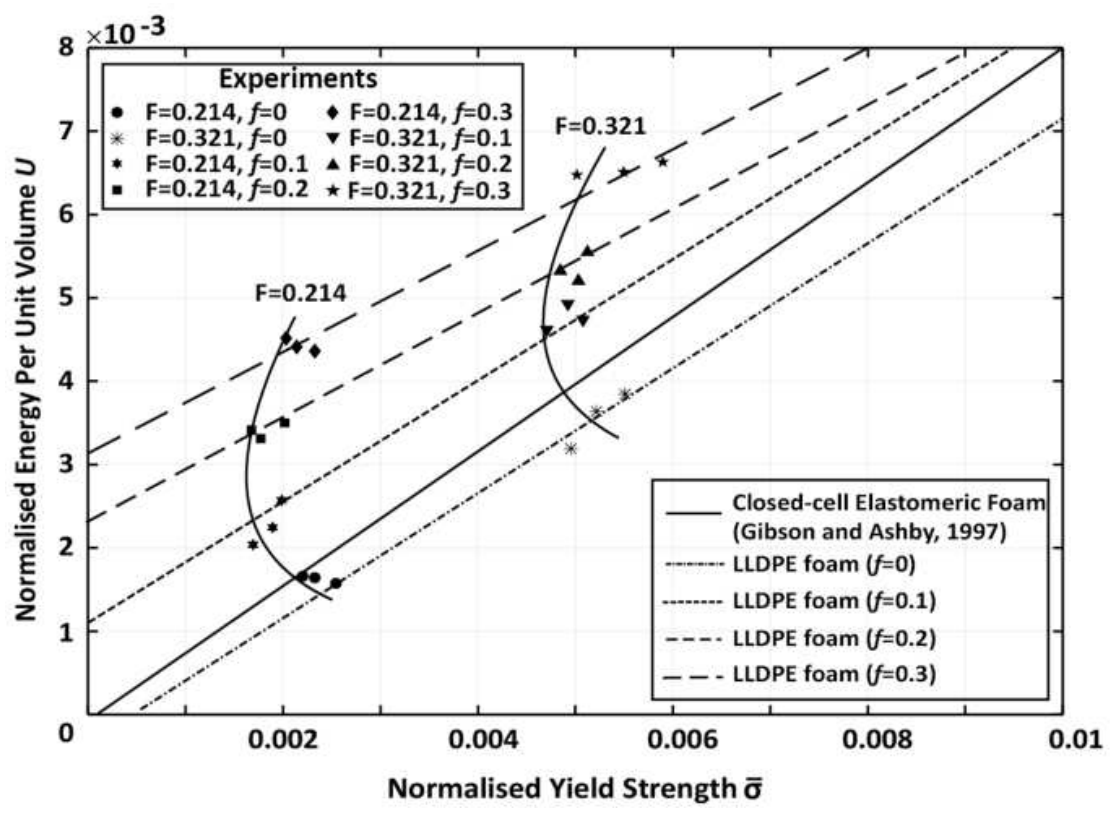

Fig 13. Comparisons of the normalised absorbed energy $U$ as a function of normalised yield strength $\bar{\sigma}$ among the pure LLDPE foams, the particle reinforced LLDPE foams and a closed-cell elastomeric foam (Gibson and Ashby, 1997).

\subsection{Finite element (FE) predictions}

Full-scale FE simulations for foam samples are computationally expensive owing to the complex internal structures. To improve the efficiency of the FE simulations, numerical study was conducted to investigate the size effect of the FE models, which aims to determine the smallest size of the FE models that can capture the behaviour of the foam samples. Let $\hat{l}$ represents the normalised edge length of the FE models, calculated as the edge length of FE models normalised by the edge length of test samples. In appendix $D$, numerical tests have been conducted to understand the effect of $\hat{l}$. It is demonstrated that (i) the FE simulation results are not sensitive to $\hat{l}$ when $0.125 \leq \hat{I} \leq 0.325$ for $f=0$, as shown in Fig. S.2 (a); (ii) stable solutions can be achieved when $\hat{l} \geq 0.25$ for $f=0.1$, as shown in Fig. S.2 (b) or $\hat{l} \geq 0.35$ for $f=0.2$, as shown in Fig. S.2 (a), and these solutions have reasonable agreement with experimental measurements. Therefore, normalised edge length of $\hat{l} \geq 0.25$ and $\hat{l} \geq 0.35$ are chosen in the FE simulations for $f \leq 0.1$ and $f=0.2$, respectively. Figures 14 (a) and (b) show the comparisons between the measured 
compressive responses and the corresponding numerical predications. The agreements are good in both the elastic region and the subsequent hardening plateau region. However, it appears that the reduced scale FE models may slightly overestimate the compressive stress, especially, when $\varepsilon>0.4$. Significant mesh distortion may occur when compressive strain $\varepsilon>0.5$, potentially resulting in the termination of the FE calculations. Hence, Fig. 14 only presents the numerical results for $\varepsilon \leq 0.5$.

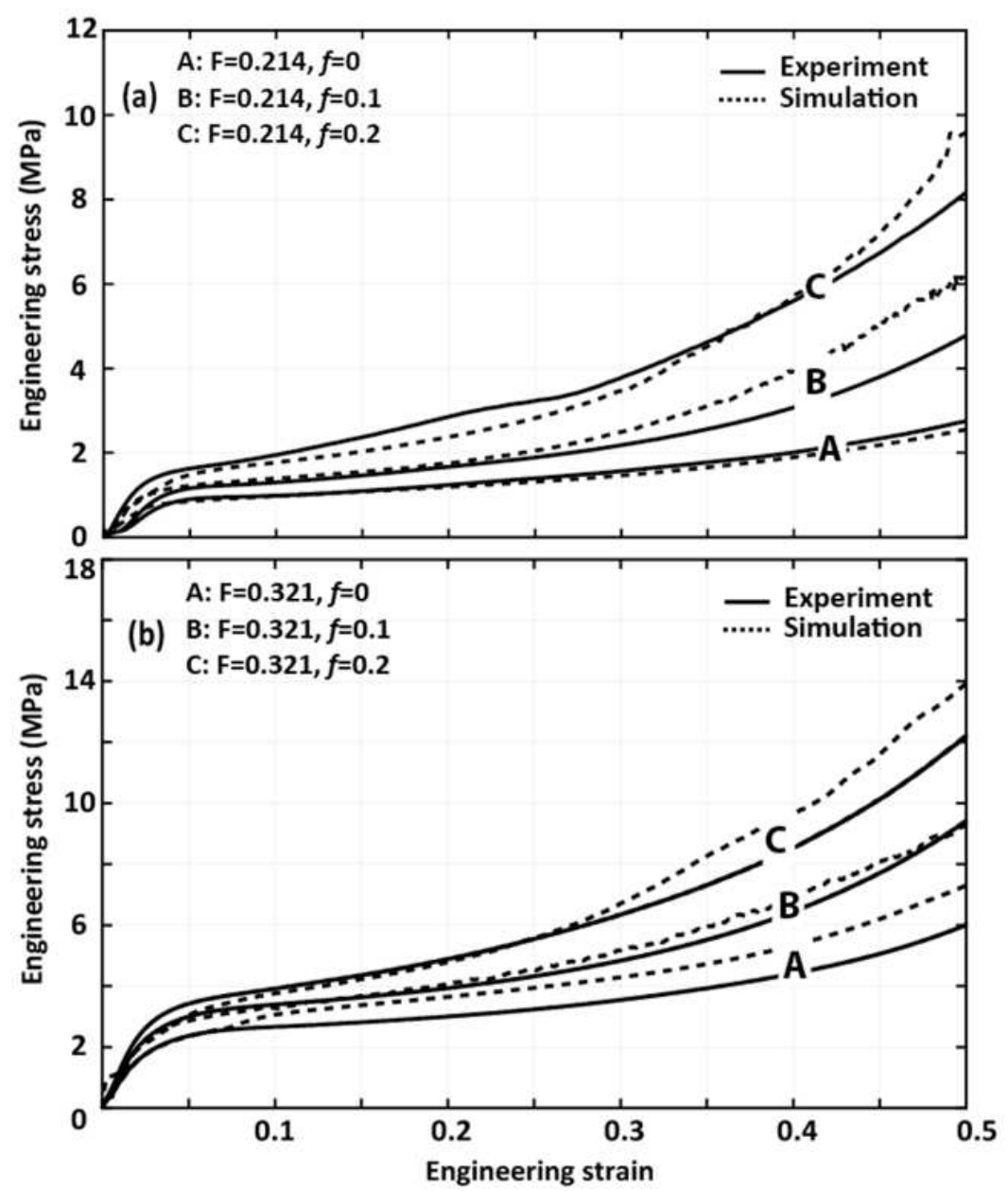

Fig 14. Comparisons of engineering stress as a function of engineering strain between the numerical predictions and experimental measurements at selected volume fractions $f$ for (a) $\mathrm{F}=0.214$, (b) $\mathrm{F}=0.321$.

\subsubsection{The predicted compressive response}

As the LLDPE foams with different polymer volume fractions have similar deformation mechanisms, the numerical results for LLDPE foams with polymer volume fraction $F=0.321$ are 
taken as representatives. For visualisation purpose, a section (E-F-G-H) is taken from each 3D model to show deformations at four selected compressive strains, i.e. $\varepsilon=0$ (initial state), $\varepsilon=0.04$ (elastic region), $\varepsilon=0.3$ (plateau region) and $\varepsilon=0.5$ (onset of densification). These are shown in Figs. 15, 16 and 17 with the contours representing the equivalent plastic strain $\bar{\varepsilon}^{p}$ within the foam matrix. Here, the equivalent plastic strain can be calculated as $\bar{\varepsilon}^{p}=\int_{0}^{t} \dot{\bar{\varepsilon}}^{p} d t$, with $\dot{\bar{\varepsilon}}^{p}$ being the equivalent plastic strain rate that is the plastic work rate conjugate to equivalent stress $\hat{\sigma}$, i.e. $\hat{\sigma} \dot{\bar{\varepsilon}}^{p}=\boldsymbol{\sigma}: \dot{\boldsymbol{\varepsilon}}^{p l}$, where $\boldsymbol{\sigma}$ and $\dot{\boldsymbol{\varepsilon}}^{p l}$ are the Cauchy stress tensor and rate of deformation tensor under plastic deformation, respectively.

Figure 15 shows the compressive response of the foam sample with $\mathrm{F}=0.321$ and $f=0$ (i.e. a pure foam). The corresponding stress-strain relation can be found in Fig. 14 (b) (Curve A). Under compressive strain $\varepsilon=0.3$ and $\varepsilon=0.5$, the geometry of the hollow cells changes from a spherical shape to an oblate spheroidal shape with strain localisation at the ends along the long axis of the spindles. Recall the matrix foam exhibits strain hardening in the plateau region with onset of densification at a compressive strain around 0.55 (Fig.9 (a) for $F=0.428$ ). The matrix foam is densified at high strain localisation region (e.g. see the green regions in Fig. $15 \varepsilon=0.04$ ). For $\varepsilon=0.5$, the top and bottom sides of the oblate spheroidal shaped cells are nearly in contact with each other, which suggests the sample is subjected to densification.

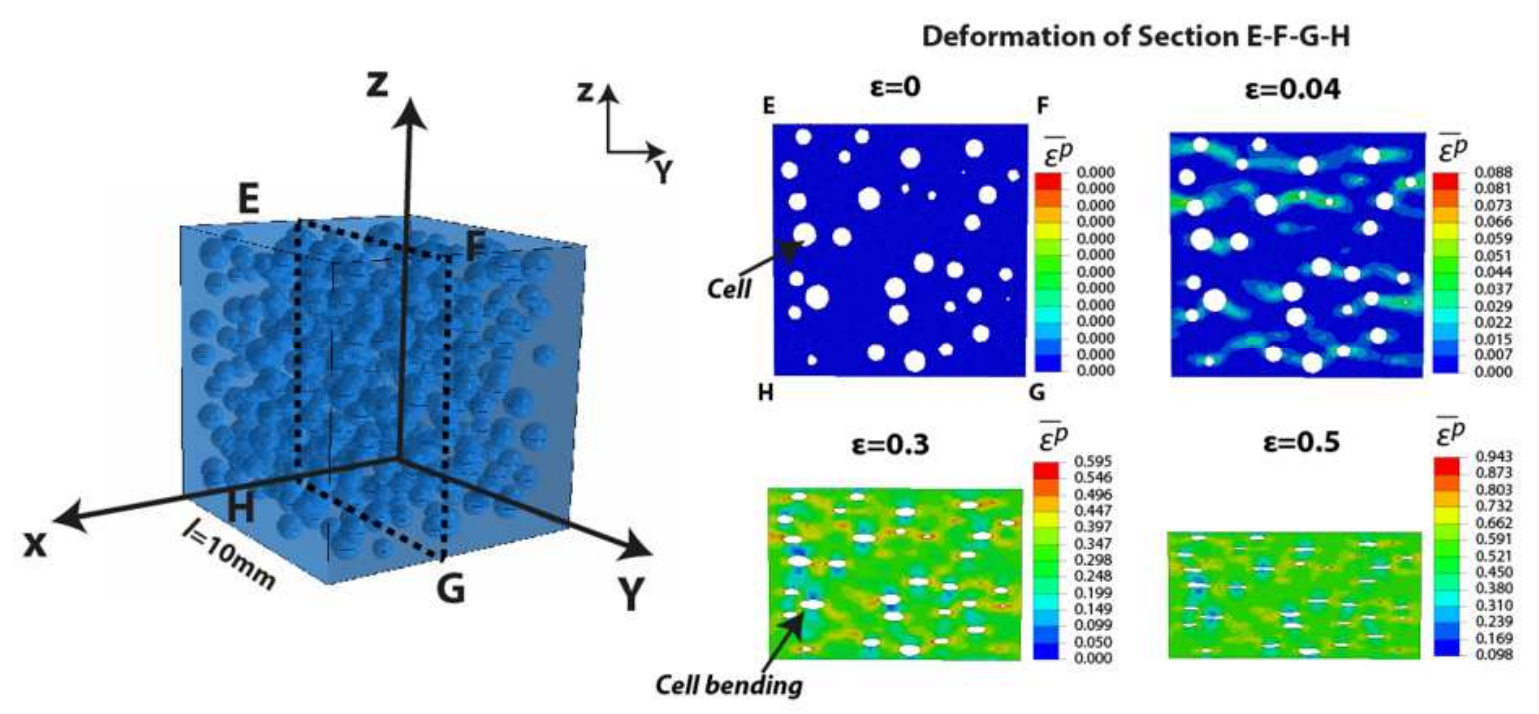


Fig 15. The compressive response of the pure LLDPE foam obtained from the FE simulations. (For interpretation of the color legend in this figure, the reader is referred to the web version of this article.)

Figures 16 and 17 show the compressive response of particle reinforced foams with $f=0.1$ and $f=0.2$, respectively. The corresponding stress-strain relations are shown in Fig. 14 (b) (Curves B and C). As shown in Figs. 16 (a) and 17 (a), strain localisations are observed around particle reinforcement at compressive strains $\varepsilon=0.3$ and $\varepsilon=0.5$ (e.g. see the green region in Figs. 16 (a) and $17(a))$, i.e. the foam matrix around the particle reinforcement is densified or close to be densified while the matrix foam around the hollow cells remains undensified. The interactions between porous internal structures of foams and particle reinforcements suggests that the hollow cells are stabilised by particle reinforcements under compression. At compressive strain $\varepsilon=0.5$, the debonding between the particle reinforcements and the foam matrix and the cracking of particles are captured by the FE simulations, see comparison between experimental observations and FE simulations, as shown in Figs. 16 (b) and 17(b). 


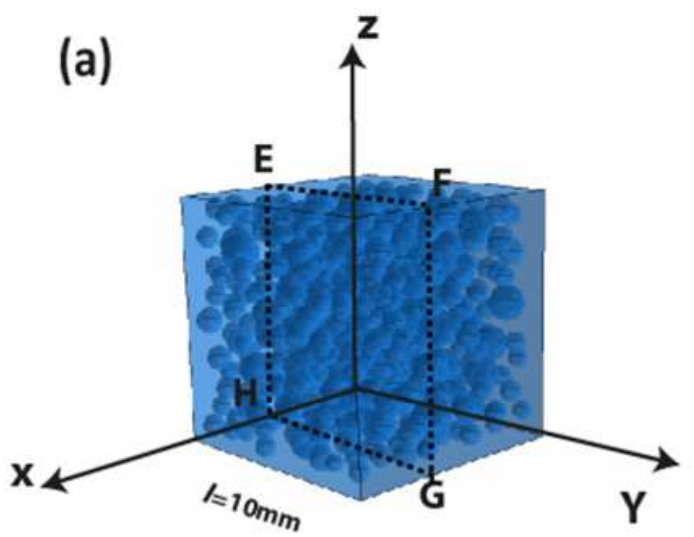

Deformation of Cross Section E-F-G-H
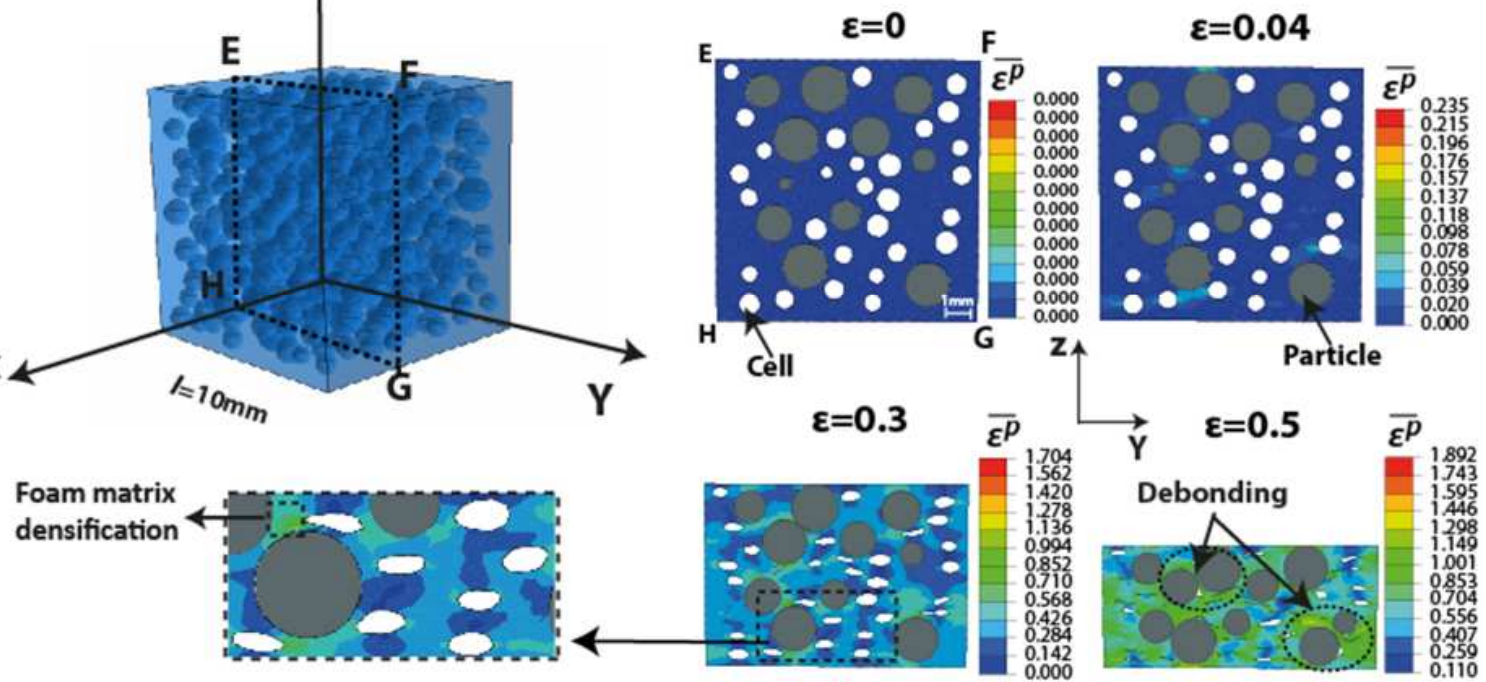

(b)
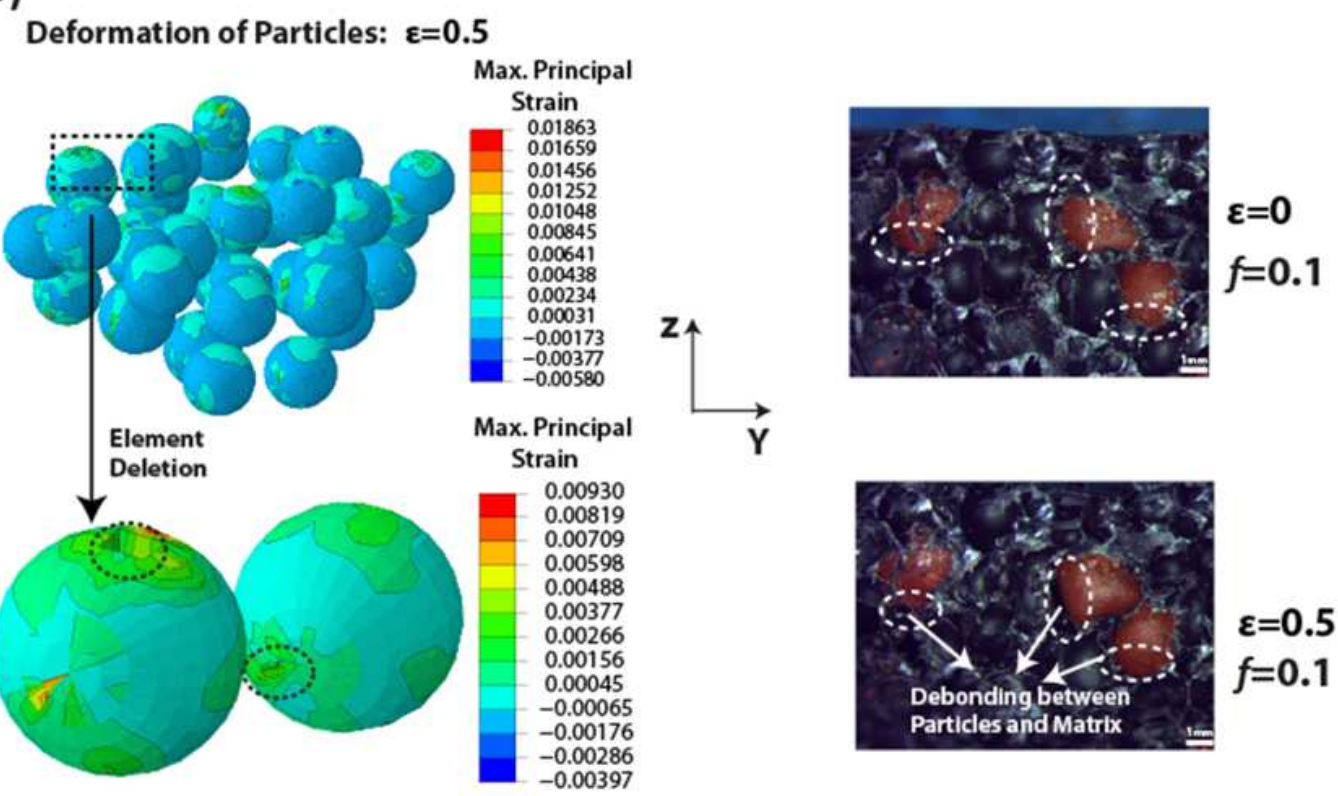

Fig 16. The compressive response of the particle reinforced LLDPE foam with $\mathrm{F}=0.321, f=0.1$ obtained from the FE simulations. (For interpretation of the color legend in this figure, the reader is referred to the web version of this article.) 

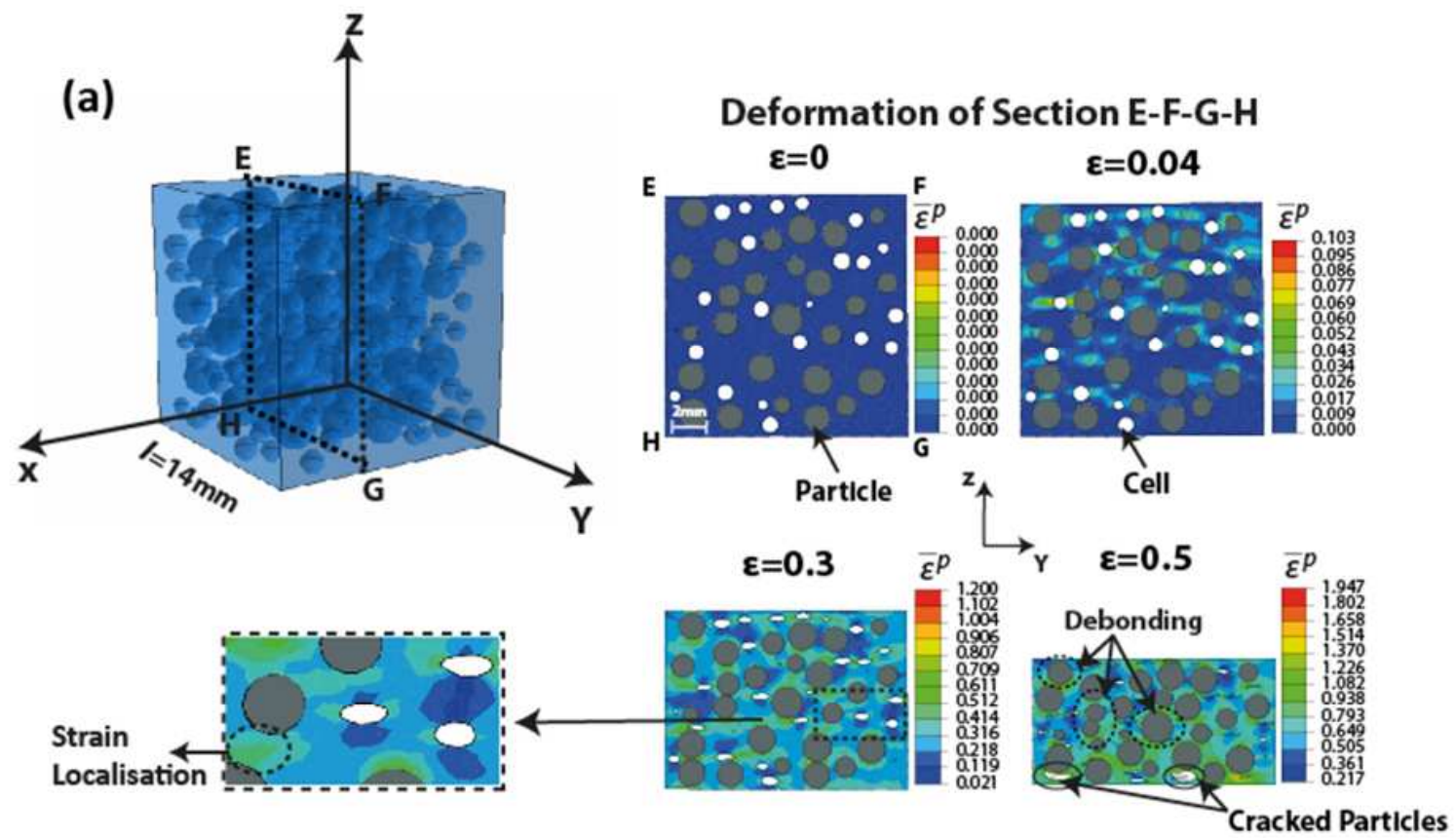

(b)

Deformation of Particles: $\boldsymbol{\varepsilon}=\mathbf{0 . 5}$

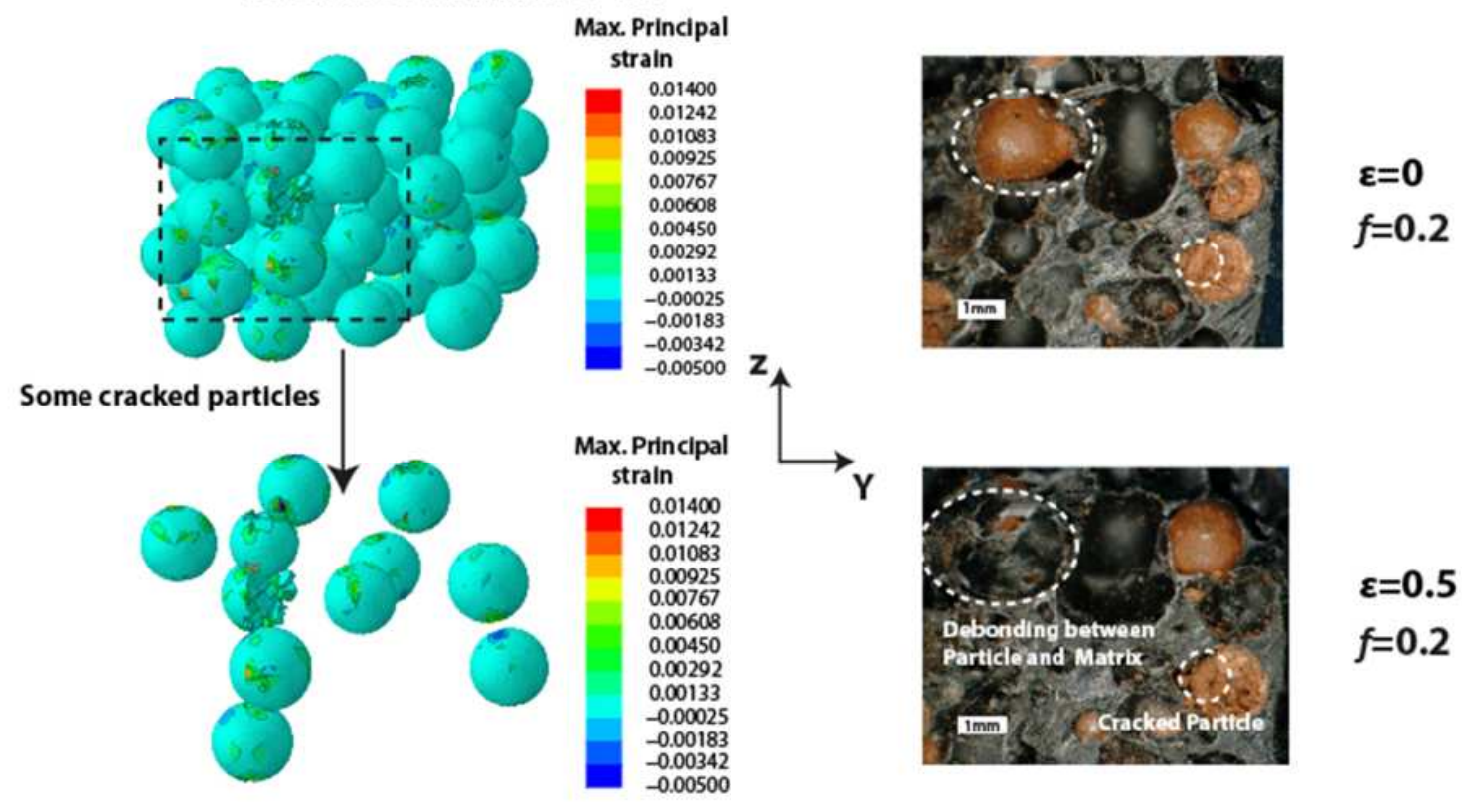

Fig 17. The compressive response of the particle reinforced LLDPE foam with $\mathbf{F}=0.321, f=0.2$ obtained from the FE simulations. (For interpretation of the color legend in this figure, the reader is referred to the web version of this article.) 


\subsubsection{The size effect of particle reinforcement and hollow cells}

\section{The particle reinforcement}

The diameter of the particle reinforcement may play an important role in the mechanical behaviour of the particle reinforced foams. To understand this role, a numerical study was conducted for the particle reinforced foam samples with $\mathrm{F}=0.214$ and $f=0.1$. The particle reinforcements employed in the simulations followed the normal distribution $d_{s} \sim N\left(d_{s m}, \gamma_{s m}^{2}=0.095 \mathrm{~mm}^{2}\right)$, with mean particle diameters $d_{s m}=0.85,1.0,1.4,1.7$ and $2.0 \mathrm{~mm}$ respectively. Recall the measured mean particle diameter is $d_{p m}=1.7 \mathrm{~mm}$, these correspond to normalised diameter of particles $d_{0}=d_{s m} / d_{p m}=0.5,0.588,0.823,1.0$ and 1.176 respectively. The FE numerical simulation results are shown in Fig. 18 for comparison. These numerical results suggest Young's modulus and yield strength of the particle reinforced foam are not very sensitive to these selected particle diameters, which may suggest the stabilisation effect is similar among these selected particle diameters. However, the particle diameter has certain effect on material hardening and densification. 


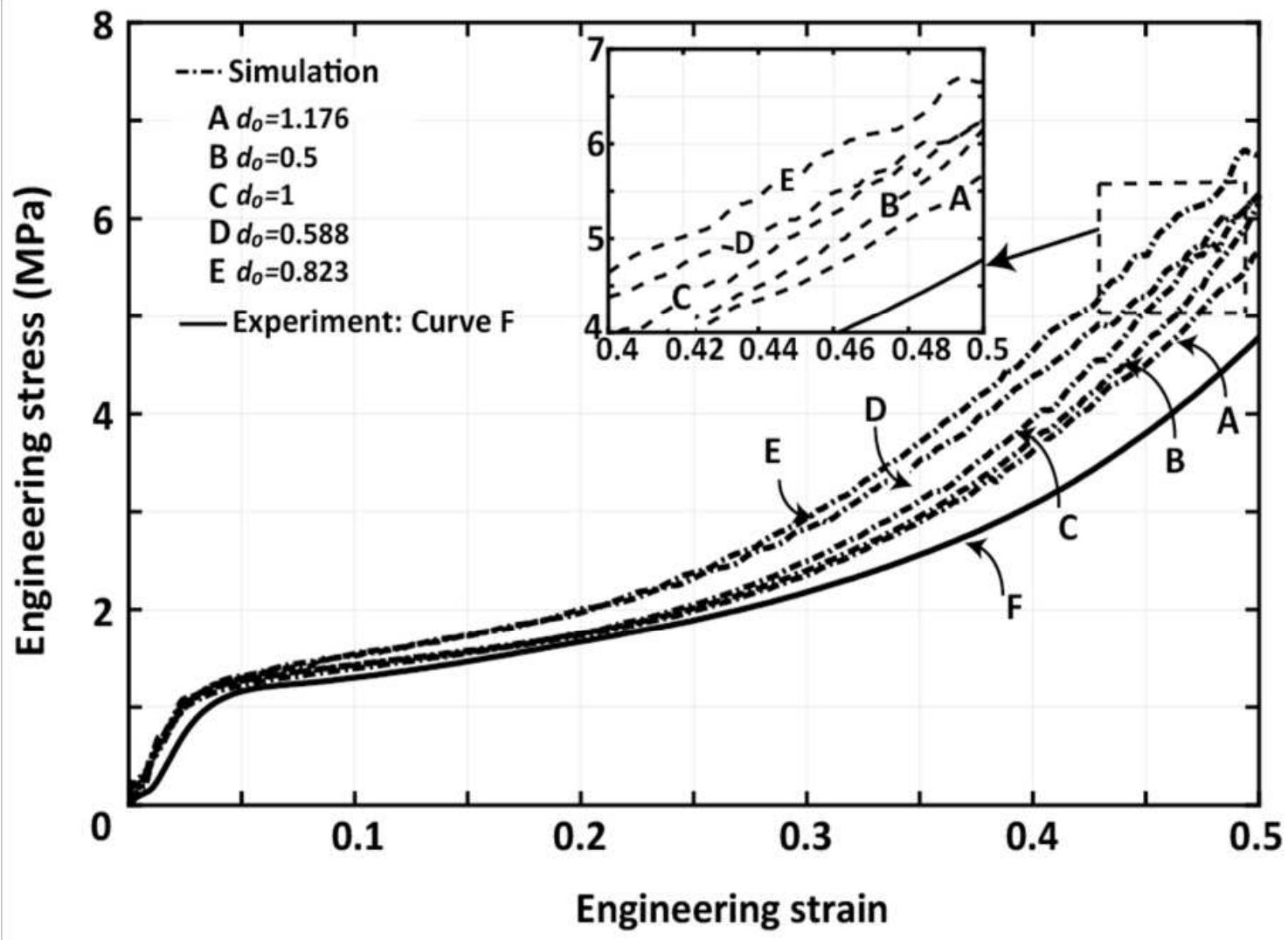

Fig 18. Size effect of particle reinforcement. The functional relations of engineering stress and engineering strain were compared between experimental measurement and simulations with $\mathrm{F}=0.214 f=0.2$. Selected normalised diameters of particles, i.e. $\mathrm{d}_{0}=\mathrm{d}_{\mathrm{sm}} / \mathrm{d}_{\mathrm{pm}}=0.5,0.588,0.823,1.0$ and 1.176 , were employed in the numerical study to evaluate the size effects of particle reinforcements.

\section{The hollow cells}

To understand the size effect of hollow cells within the foam structure, a numerical study was conducted on the pure foam sample with volume fraction $\mathrm{F}=0.214$ as being representative. Recall the FE model of the foam sample was generated via inserting the hollow cells with diameters following the normal distribution $D_{c} \sim N\left(D_{c m}=3.0 \mathrm{~mm}, \gamma_{c m}^{2}=0.16 \mathrm{~mm}^{2}\right)$ into the foam matrix with $\mathrm{F}=0.267$, see Section 4.1. The following mean diameters of hollow cells were chosen in the parametric study to evaluate the size effect with $\gamma_{c m}^{2}=0.16 \mathrm{~mm}^{2}$, and $D_{c m}=1.4,2.0,3.0,4.0$ and $5.0 \mathrm{~mm}$. Recall the measured mean diameters of the hollow cells is $D_{c m}=1.752 \mathrm{~mm}$ (Figure 2), 
these correspond to normalised mean diameters of the hollow cells $D_{0}=D_{d m} / D_{c m}=0.799,1.414,1.712,2.283$ and 2.854 , respectively. The stress-strain curves obtained by the numerical study are shown in Fig. 17. The stress-strain curve of the pure foam with $F=0.267$ (Curve A) is shown in the figure for comparison. The results demonstrate that Young's modulus, yield strength and stress in hardening decrease with increase of the mean cell diameters. However, the size effect is less significant compared to the volume fraction effect $F$ (see the Curve A with $\mathrm{F}=0.267$ in Fig. 19).

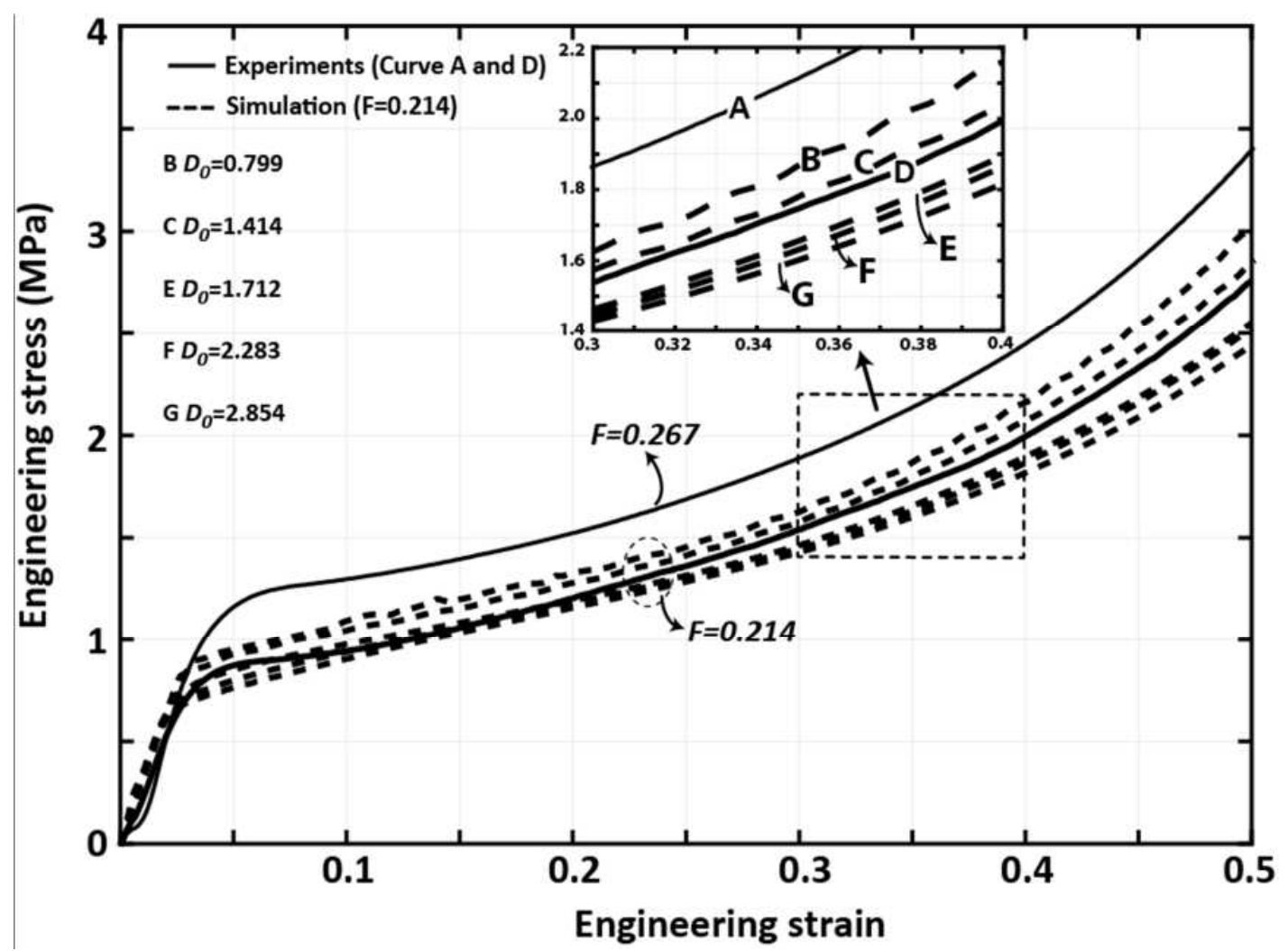

Fig 19. Size effect of the hollow cells. The functional relations of engineering stress and engineering strain were compared between experiments $(\mathrm{F}=0.214 f=0$ and $\mathrm{F}=0.267 f=0)$ and simulations ( $\mathrm{F}=0.214 f=0$ ). The selected normalised diameter of hollow cells, i.e. $D_{0}=D_{d m} / D_{c m}=0.799,1.414,1.712,2.283$ and 2.854 , were employed in the numerical study to evaluate the size effects of hollow cells. 


\section{Concluding remarks}

The quasi-static compressive responses of LLDPE foams with and without particle reinforcement have been investigated through experiments, theoretical analysis and finite element simulations. The internal structures of the foam samples were characterised by optical microscopes and X-ray CT scanning. Compression tests were conducted using the procedure defined by ASTM 1621-04a. To capture the mechanism of deformation, the finite element simulations explicitly modelled the randomly distributed hollow cells and particle reinforcement within a foam sample based on the observed internal structures.

Experimental measurement has demonstrated that the reinforcing effects of particle reinforcements include: (1) enhancement of elastic modulus and yield strength; (2) increased strain-hardening rate in plateau region; and (3) higher stresses in the densification region. The particle reinforced LLDPE foams exhibit higher energy absorption capacity than pure LLDPE foams, which can be explained as (1) stabilisation to bending of hollow cells within the foams prior to foam densification, and (2) particle crushing after densification occurs. Theoretical models proposed by Kernel (1956), Isai and Cohen (1967) Halpin and Tsai (1969) and Gibson and Ashby (1997) were employed to predict the yield strengths and elastic moduli of LLPDE foams. The theoretical predictions show good agreements with experimental measurements.

It has been demonstrated that reduced scale FE models can be employed to predict the compressive response of LLDPE foams with good agreements.

To reduce the computational scale, numerical study has been conducted to determine the smallest size of the FE models that can capture the behaviour of the foam samples. The FE predictions captured the interaction between particle reinforcements and porous structures of the foams: during the loading, the foam matrix around the particle reinforcement is densified or close to being densified while the foam matrix around the hollow cells remains undensified, which suggests hollow cells are stabilised by particle reinforcement. Based on the FE model, the size effects of particle reinforcement and hollow cells were studied, which suggested simulation 
results were not very sensitive to them at the selected range of particle diameters and hollow cell diameters.

To avoid full-scale numerical simulations of composite materials with periodic unit cells, Representative Volume Element (RVE) analysis has been employed by existing research (Cho et al., 2017), in which periodic boundary conditions need to be imposed to the RVE. However, owing to the stochastic nature of the internal structures of the foams, further validation is needed in order to apply RVE analysis for our current simulations.

\section{Acknowledgements}

The authors acknowledge the support from the Defence of Science and Technology Laboratory, UK (DSTL, DSTLX-1000104239) and the Royal Society (a charity registered in England with number 207043) through Research Grant Scheme. They also acknowledge the High Performance Computing facility at the University of Nottingham. The first author would like to acknowledge the financial support of China Scholarship Council (CSC). 


\section{APPENDIX}

\section{A. Creating the FE model of pure foam with $F=0.214$}

The modelling strategy can be schematically shown in Fig. S.1 for the creation of the FE model for the pure foam samples with $F=0.214$. The pure foam with $F=0.214$ has the measured cells diameter range of $0.464 \mathrm{~mm}$ to $3.824 \mathrm{~mm}$, and follows $D_{c} \sim N\left(D_{c m}=1.752 \mathrm{~mm}, \gamma_{c m}^{2}=0.641 \mathrm{~mm}^{2}\right)$; the pure foam with $\mathrm{F}=0.267$ has the measured cells diameter range of $0.659 \mathrm{~mm}$ to $2.356 \mathrm{~mm}$, and follows $D_{c} \sim N\left(D_{c m}=1.282 \mathrm{~mm}, \gamma_{c m}^{2}=0.381 \mathrm{~mm}^{2}\right)$. Hence, the FE model with $\mathrm{F}=0.214$ was created via inserting hollow cells with diameter range of $2.120 \mathrm{~mm}$ to $3.660 \mathrm{~mm}$ and following $D_{c} \sim N\left(D_{c m}=2.9, \gamma_{c m}^{2}=0.25\right)$ into the foam matrix $(F=0.267)$.

(a)

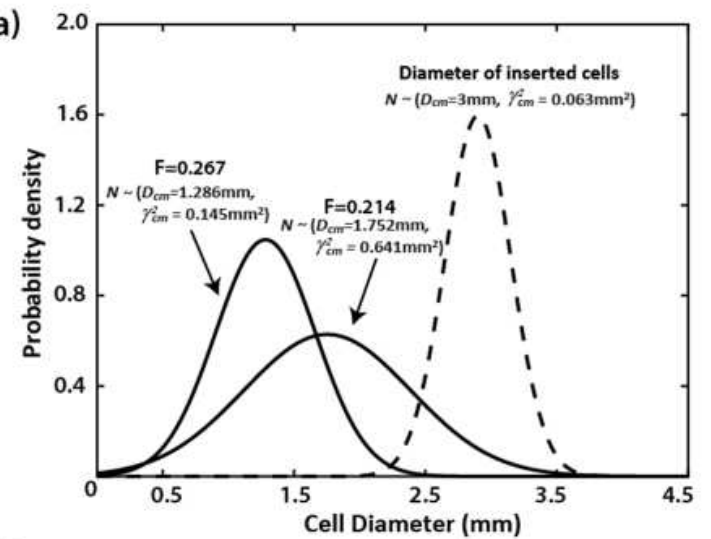

(b)

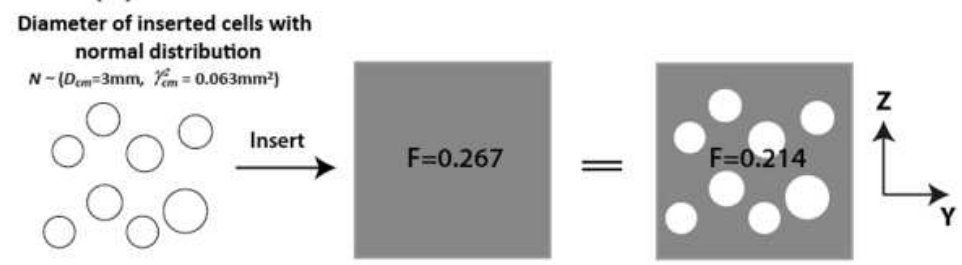

Fig S.1. Creation of the numerical models for pure foams with $F=0.214$. (a) The distributions of cells diameter for the pure foams with $F=0.214$ and $F=0.267$ as well as the inserted cells. (b) The hollow cells which follow $D_{c} \sim N\left(D_{c m}=2.9, \gamma_{c m}^{2}=0.25\right)$ were inserted to the foam matrix with $\mathrm{F}=0.267$ to create the FE model of the pure foam with $F=0.214$. (For interpretation of the color legend in this figure, the reader is referred to the web version of this article.) 


\section{B. Theoretical analysis of yield strength}

Pure LLDPE foams

Gibson and Ashby (1997) suggests that yield strength $\sigma_{f}$ can be related to density of the foam $\rho_{f}$ and gas pressure within the cells, shown as

$$
\frac{\sigma_{f}}{E_{s}} \approx 0.05\left(\frac{\rho_{f}}{\rho_{s}}\right)^{2}+\frac{\left(p_{0}-p_{a t}\right)}{E_{s}}
$$

where $E_{s}$ the elastic modulus of LLDPE in dense solid form, $p_{0}$ the gas pressure within in cells and $p_{a t}$ the atmospheric pressure. For man-made foams, $p_{0}=p_{a t}$ (Gibson and Ashby, 1997). Then, Eq. (A.1) can be written as

$$
\frac{\sigma_{f}}{E_{s}} \approx 0.05\left(\frac{\rho_{f}}{\rho_{s}}\right)^{2}
$$

\section{Particle reinforced LLDPE foams}

Let $\sigma_{c}$ represents the yield strength of composites, i.e. the particle reinforced foam in this study, which consists of particle reinforcement and foam matrix. $\sigma_{c}$ can be approximated by the inverse rule of mixtures, i.e. the Reuss lower bound (Lakes and Drugan, 2002), described in Eq. (A. 3). Inverse rule of mixtures is based on the assumption that the stress acting on the reinforcement equates the stress acting on the matrix.

$$
\sigma_{c}=\frac{\sigma_{p} \sigma_{f}}{f \sigma_{f}+(1-f) \sigma_{p}}
$$

where $\sigma_{p}$ and $f$ denote the yield strength and the volume fraction of the particle reinforcement, respectively, $\sigma_{f}$ the yield strength of LLDPE foam matrix. Substituting Eq. (A.3) through (A.2), the yield strength of a particle reinforced foam can be approximated as 


$$
\sigma_{c}=\frac{0.05 \sigma_{p} E_{s}\left(\frac{\rho_{f}}{\rho_{s}}\right)^{2}}{0.05 f E_{s}\left(\frac{\rho_{f}}{\rho_{s}}\right)^{2}+(1-f) \sigma_{p}}
$$

The experimental measurement has a good agreement with the prediction obtained by Eq. (A.2), as shown in Fig.9 (b). For pure foam, relative density $\tilde{\rho}=\rho_{f} / \rho_{s}$ equates volume fraction $F$. Hence, yield strength $\sigma_{f}$ scales with $\mathrm{F}^{2}$. For particle reinforced foams, the experimental measurement of yield strength $\sigma_{c}$ as the function of $f$ has a good agreement with the prediction by the Reuss lower bound (Eq. (A. 4)) except for the results when $f \geq 0.3$, as shown in Fig.10 (c).

\section{Theoretical analysis of elastic modulus}

Pure LLDPE foams

Gibson and Ashby (1997) suggests that the elastic modulus of a pure foam $E_{f}$ can be expressed as the function of constants $C_{1}$ and $C_{1}$ which relate to the geometry of hollow cells, elastic modulus of solid LLDPE $E_{s}$, relative density $\tilde{\rho}$ and fraction of solid contained in separation $\varphi$ (Gibson and Ashby, 1997), shown as

$$
\frac{E_{f}}{E_{s}}=C_{1} \varphi^{2} \tilde{\rho}^{2}+C_{1}^{\prime}(1-\varphi) \tilde{\rho}
$$

Assuming $\varphi$ is a constant value that is not sensitive to relative density of foams, $C_{1}$ and $C_{1}^{\prime}$ can be obtained through curve fitting against the experimentally measured elastic moduli, see Fig. 9 (c). Eq. (B, 1) can be rewritten as

$$
\frac{E_{f}}{E_{s}}=0.9565 \tilde{\rho}^{2}+0.186 \tilde{\rho}=0.9565 \mathrm{~F}^{2}+0.186 \mathrm{~F}
$$




\section{Particle reinforced LLDPE foams}

Kernel (1956), Isai and Cohen (1967) and Halpin and Tsai (1969) have proposed theoretical models to predict the elastic modulus of a particle reinforced composite. The basic Kernel's model gives in

with

$$
\begin{gathered}
E_{c}=E_{f}\left(\frac{1-A B f}{1-B f}\right) \\
A=\frac{7-5 v}{8-10 v} \\
B=\frac{E_{p} / E_{f}}{E_{p} / E_{f}+A}
\end{gathered}
$$

Here, $E_{c}, E_{f}$ and $E_{p}$ are the elastic modulus of the particle reinforced foam, the foam matrix and the particle reinforcement, respectively. $v$ is the Poisson's ratio of LLDPE foam matrix which has the value of 0 . Ishai and Cohen extended the Kernel's model to account for uniform displacement at the boundary, which reads (Yang et al., 2004; Isai and Cohen, 1967)

$$
E_{c}=E_{f}\left[1+\frac{f}{m /(m-1)-f^{1 / 3}}\right]
$$

with

$$
m=E_{p} / E_{f}
$$

Halpin and Tsai (1969) considered the micromechanics analyses to approximate the results.

$$
E_{c}=E_{f} \frac{1+\delta \eta f}{1-\eta f}
$$

where

$$
\eta=\frac{E_{p} / E_{f}-1}{E_{p} / E_{f}+\delta}
$$

$\delta$ is the non-dimensional number decided by the geometry of filler. For spherical particles, $\delta=2$ (Yang et al., 2004). Fig. 10 (d) shows that the predictions obtained from theoretical models are in good agreements with experimental measurements. 


\section{Size effect of the FE model}

To determine the dimension of reduced scale models which can provide converged results, FE models with different dimension were created for calculation. Let $\hat{l}$ represents the normalised edge length of the FE models, calculated as the edge length of FE models normalised by the edge length of test samples. Figure S.2 shows the comparisons between experimental measurements and FE predictions with selected values of $\hat{l}$ with $\mathrm{F}=0.321$. The $\mathrm{FE}$ simulation results are not sensitive to $\hat{l}$ when $0.125 \leq \hat{I} \leq 0.325$ with $f=0$ and the reduced scale models can offer stable solutions when $\hat{l} \geq 0.25$ with $f=0.1$ or $\hat{l} \geq 0.35$ with $f=0.2$. These solutions have reasonable agreements with experimental measurements. Therefore, normalised edge length of $\hat{I} \geq 0.25$ and $\hat{l} \geq 0.35$ were chosen in the FE simulations for $f \leq 0.1$ and $f=0.2$, respectively. 


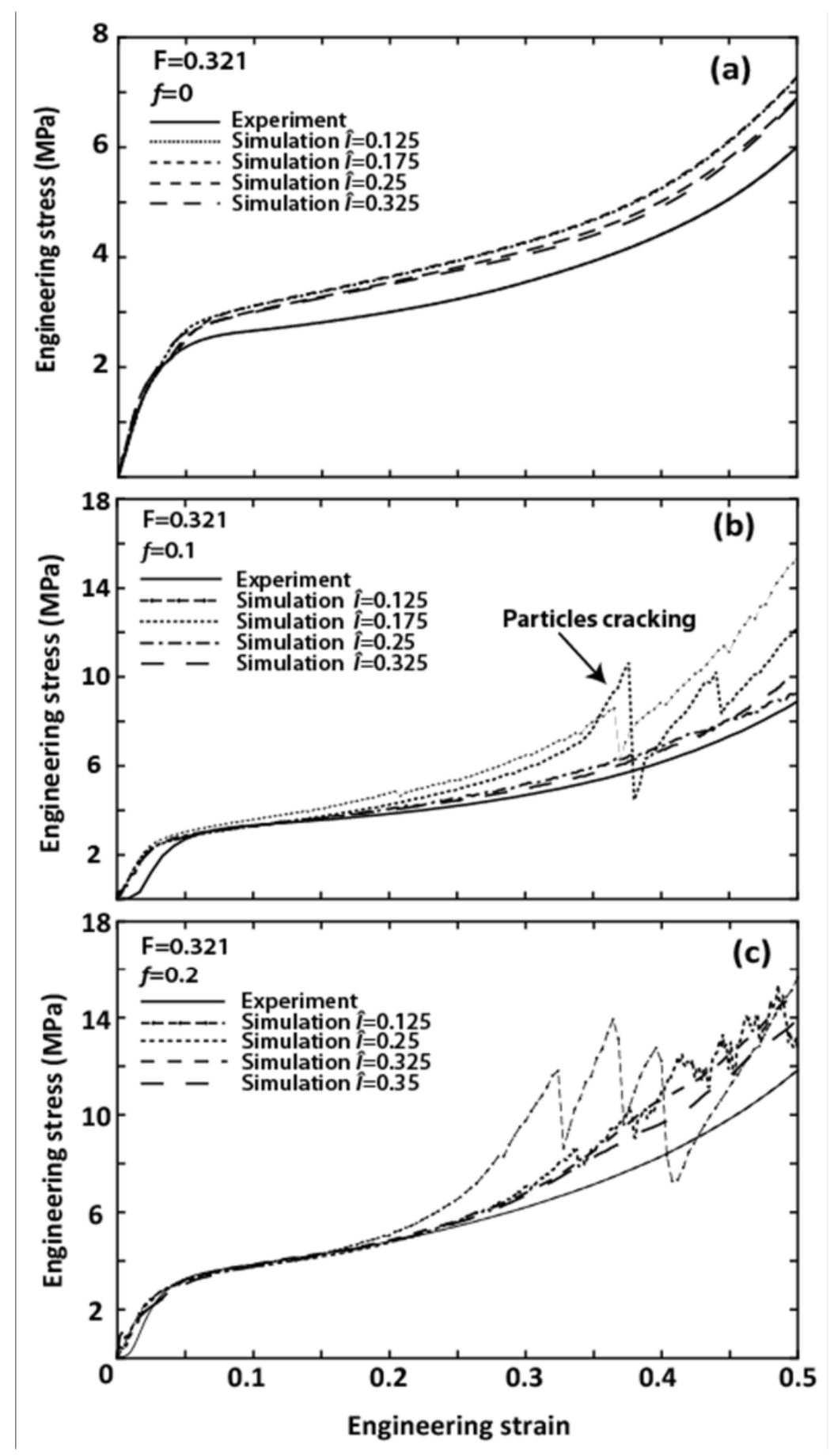

Fig S.2. Scale effects of the FE models. The relations of engineering stress and engineering strain of the LLDPE foams with $\mathrm{F}=0.321 f=0, \mathrm{~F}=0.321 f=0.1$ and $\mathrm{F}=0.321 f=0.2$ were compared between simulations obtained from reduced scale FE models with selected values of $\hat{l}$ and experimental measurement. 


\section{References}

ASTM, 2018. ASTM D1621-16 standard test method for compressive properties of rigid cellular plastics.

Dassault Systemes, ABAQUS User's \& Theory Manuals - Release 6.13-1. Providence, RI, USA; 2013.

Ashby. M.F., 1993. Criteria for selecting the components of composites. Acta Mater. 41, 13131335. https://doi.org/10.1016/0956-7151(93)90242-K.

Ashby. M.F., 2006. The properties of foams and lattices. Philos. T. R. Soc. A. 364, 15-30. https://doi.org/10.1098/rsta.2005.1678.

Brown. K.A., Brooks. R., Awoyemi. O, 2011. Meso-scale modelling of shock wave propagation in a cellular glass particle reinforced thermoplastic composite. $18^{\text {th }}$ International Conference of Composites Materials.

Chawla. N., Chawla. K. K., 2006. Microstructure-based modelling of the deformation behaviour of particle reinforced metal matrix composites. J. Mater. Sci. 41 (3), 913-925. https://doi: 10.1007/s10853-006-6572-1.

Chen. Y.M., Das. R., Battley. M., 2015. Effects of cell size and cell wall thickness variations on the stiffness of closed-cell foams. Int. J. Solids Struct. 52, 150-164. https://doi.org/10.1016/j.ijsolstr.2014.09.022.

Cho. Y.J., Lee. W., Park. Y.H., 2017. Finite Element Modeling of Tensile Deformation Behaviors of Iron Syntactic Foam with Hollow Glass Microspheres. Materials. 10(10), 1201- 1216. https://doi: 10.3390/ma10101201.

Dahmani. L., Mohand. C.S.H., 2011. Modélisation and influence of shear retention parameter on the response of cast iron beam. Procedia Engineering. 10, 19-24. https://doi.org/10.1016/j.proeng.2011.04.005.

Deshpande. V.S., Fleck. N.A., 2000. Isotropic constitutive models for metallic foams. J. Mech. Phys. Solids. 48(6-7), 1253-1283. https://doi.org/10.1016/S0022-5096(99)00082-4.

Evans. A.G., He. M.Y., Deshpande. V.S., Hutchinson. J.W., Jacobsen. A.J., Carter. W.B., 2010. Concepts for enhanced energy absorption using hollow micro-lattices. Int. J. Impact Eng. 37(9), 947-959. https://doi.org/10.1016/j.ijimpeng.2010.03.007.

Esmaeelzadeh. S., Simchi. A., Lehmhus. D., 2006. Effect of ceramic particle addition on the foaming behavior, cell structure and mechanical properties of P/M AlSi7 foam. Ma.t Sci. Eng. A- Struct. 424(1-2), 290-299. https://doi.org/10.1016/j.msea.2006.03.013.

Fischer. F., Lim. G.T., Handge. U.A., Altstabt. V., 2009. Numerical Simulation of Mechanical Properties of Cellular Materials Using Computed Tomography Analysis. J. Cell Plast. 45, 441460. https://doi.org/10.1177/0021955X09339340.

Gibson, L.J., Ashby, M.F., 1999. Cellular solids: structure and properties. Cambridge University Press.

Gupta. N., Pinisetty. D., Shunmugasamy. V.C., 2013. Reinforced Polymer Matrix Syntactic Foams: Effect of Nano and Micro-Scale Reinforcement. Springer.

Halpin. J.C., Tsai. S.W., 1969. Effects of Environmental Factors on Composite Materials. Air Force Technical Report AFML-TR, 67-423. 
Hillerborg. A., Modeer. M., Petersson. P.E., 1976. Analysis of Crack Formation and Crack Growth in Concrete by Means of Fracture Mechanics and Finite Elements. Cement Concrete Res. 6(6), 773-781. https://doi.org/10.1016/0008-8846(76)90007-7.

Ishai. O., Cohen. L.J., 1967. Elastic Properties of Filled and Porous Epoxy Composites. Int. J Mech. Sci. 9(8), 539-546. https://doi.org/10.1016/0020-7403(67)90053-7.

Jayavardhan. M.L., Doddamani. M, 2018. Quasi-static compressive response of compression molded glass microballoon/HDPE syntactic foam. Compos. Part B-Eng. 149, 165-177. https://doi.org/10.1016/j.compositesb.2018.04.039.

Kerner. E.H., 1956. The Electrical Conductivity of Composite Media. Proc. Phys. Soc. B. 69(8), 802807. https://doi: 10.1088/0370-1301/69/8/304.

Lakes. R.S., Drugan. W.J., 2002. Dramatically stiffer elastic composite materials due to a negative stiffness phase. J Mech. Phys. Solids. 50(5), 979-1009. https://doi.org/10.1016/S00225096(01)00116-8/.

Li. Y.C., Xiong. J.Y., Lin. J.G., Forrest. M., Hodgson. P.D., Wen. C.E., 2007. Mechanical properties and energy absorption of ceramic particulate and resin-impregnation reinforced aluminium foams. Materials Forum Volume 31.

Liu. J., Yu. S., Zhu. X., Wei. M., Luo. Y., Liu. Y., 2009. Correlation between ceramic additions and compressive properties of $\mathrm{Zn}-22 \mathrm{Al}$ matrix composite foams. J. Alloys. Compd. 476(1-2), 220225. https://doi.org/10.1016/j.jallcom.2008.09.069.

Liu. Y., Gong. X.L., 2006. Compressive behavior and energy absorption of metal porous polymer composite with interpenetrating network structure. T. Nonferr. Metal. Soc. 16 (2), s439-s443. https://doi.org/10.1016/S1003-6326(06)60229-X.

Maiti. S.K., Gibson. L.J., Ashby. M.F., 1984. Deformation and Energy Absorption Diagrams for Cellular Solids. Acta Mater. 32(11), 1963-1975. https://doi.org/10.1016/0001-6160(84)901779.

Marvi-Mashhadi. M., Lopes. C.S., LLorca. J., 2018. Effect of anisotropy on the mechanical properties of polyurethane foams: An experimental and numerical study. Mech. Mater. 124, 143-154. https://doi.org/10.1016/j.mechmat.2018.06.006.

Marvi-Mashhadi. M., Lopes. C.S., LLorca. J., 2018. Modelling of the mechanical behaviour of polyurethane foams by means of micromechanical characterization and computational

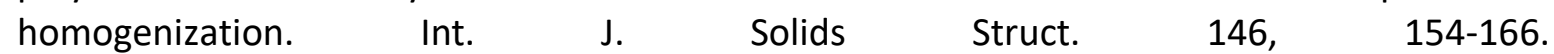
https://doi.org/10.1016/j.ijsolstr.2018.03.026.

Mondal. D.P., Goel. M.D., Das. S, 2009. Compressive deformation and energy absorption characteristics of closed cell aluminium-fly ash particle composite foam. Ma.t Sci. Eng. AStruct. 507(1-2), 102-109. https://doi.org/10.1016/j.msea.2009.01.019.

Rice. M.C., Fleischer. C.A., Zupan. M., 2006. Study on the Collapse of Pin-Reinforced Foam Sandwich Panel Cores. Exp. Mech. 46(2), 197-204. https://doi.org/10.1007/s11340-006-71033.

Richardson. J.T., Peng. Y., Remue. D., 2000. Properties of ceramic foam catalyst supports: pressure drop. Appl. Catal., A. 204(1), 19-32. https://doi.org/10.1016/S0926-860X(00)005081.

Samimi. A., Hassanpour. A., Ghadiri. M., 2005. Single and bulk compressions of soft granules: Experimental study and DEM evaluation. Chem. Eng. Sci. 60(14), 3993-4004. https://doi.org/10.1016/j.ces.2005.02.036. 
Srinivasa. P., Kulachenko. A., 2015. Analysis of the compressive response of Nano Fibrillar Cellulose foams. Mech. Mater. 80, 13-26. https://doi.org/10.1016/j.mechmat.2014.09.006.

Thornton. C., Ning. Z., 1998. A theoretical model for the stick/bounce behaviour of adhesive, elastic-plastic spheres. Powder Technol. 99(2), 154-162. https://doi.org/10.1016/S00325910(98)00099-0.

Verdolotti. L., Lavorgna. M., Lamanna. R., Maio. E.D., lannace. S., 2015. Polyurethane-silica hybrid foam by solegel approach: Chemical and functional properties. Polymer. 56, 20-28. https://doi.org/10.1016/j.polymer.2014.10.017.

Wichianrat. E., Boonyongmaneerat. Y., Asavavisithchai. A, 2012. Microstructural examination and mechanical properties of replicated aluminium composite foams. T. Nonferr. Metal Soc. 22(7), 1674-1679. https://doi.org/10.1016/S1003-6326(11)61372-1.

Yu. W., Qian. M., Liang. X., Li. H. J., 2016, Numerical estimation of effective elastic moduli of syntactic foams reinforced by short glass fibres. Ceram.-Silikaty. 60(2), 77-84. https://doi.org/10.13168/cs.2016.0012.

Yang. W., Shi. W., Li. Z.M., Xie. B.H., Feng. J.M., Yang. M.B., 2004. Mechanical Properties of Glass Bead-filled Linear Low-density Polyethylene. J. Elastom Plast. 36(3), 251-265. https://doi.org/10.1177/0095244304042989.

Zegeye. E.F., Woldesenbet. E., 2012. Processing and mechanical characterization of carbon nanotube reinforced syntactic foams. J. Reinf. Plast. Comp. 31(15), 1045-1052. https://doi.org/10.1177/0731684412452919. 


\section{Figure captions}

Fig 1. Compressive force as a function of the displacement of the movable crosshead of test machine obtained via single ceramic particle compression test and numerical simulation. The insert on the right schematically shows the compression test. The insert on the left shows the distribution of the diameters of the particles.

Fig 2. The distributions of the measured diameters of the hollow cells and microscopic images of the sectioned LLDPE pure foam samples with $F=0.214$ as shown in (a) and (b), $F=0.267$ as shown in (c) and (d), F = 0.321 as shown in (e) and (f) and $F=0.428$ as shown in (g) and (h), respectively. (For interpretation of the color legend in this figure, the reader is referred to the web version of this article.)

Fig 3. Microscopic images and X-ray CT images of the particle reinforced foam samples with $\mathrm{F}=0.214, f=0.1$ as shown in (a) and (c) and $\mathrm{F}=0.214, f=0.2$ as shown in (b) and (d), respectively. (For interpretation of the color legend in this figure, the reader is referred to the web version of this article.)

Fig 4. Generation of the structure of a particle reinforced foam was achieved via a 3-step procedure. (For interpretation of the color legend in this figure, the reader is referred to the web version of this article.)

Fig 5. Microscopic images of the sectioned LLDPE pure foam samples before compression for (a) $F=0.214$ and (b) $F=0.321$; and after compression with permanent compressive strain $\varepsilon=0.3$ for (c) $F=0.214$ and (d) $F=0.321$. (For interpretation of the color legend in this figure, the reader is referred to the web version of this article.)

Fig 6. Creation of the numerical models for pure foams with $F=0.321$. (a) The distributions of cells diameter for the pure foams with $F=0.321$ and $F=0.428$ as well as the inserted cells. (b) The hollow cells which follow $D_{c} \sim N\left(D_{c m}=1.56 \mathrm{~mm}, \gamma_{c m}^{2}=0.01 \mathrm{~mm}^{2}\right)$ were inserted to the foam matrix with $F=0.428$ to create the $F E$ model of the pure foam with $F=0.321$. (For interpretation of the color legend in this figure, the reader is referred to the web version of this article.) 
Fig 7. Finite element model for a cubic particle reinforced foam sample. (For interpretation of the color legend in this figure, the reader is referred to the web version of this article.)

Fig 8. (a) The relation between tensile stress and crack-opening displacement after a crack is initiated; (b) The bilinear softening model defines the relation between shear retention factor and crack opening strain.

Fig 9. (a) The uniaxial stress-strain relation of pure LLDPE foams at selected polymer volume fraction $\mathrm{F}$. (b) The relation of yield strength $\sigma_{f}$ and volume fraction Ffor pure LLDPE foams is compared between experimental measurements and theoretical predictions obtained from Gibson and Ashby model (1997). (c) Elastic modulus $E_{f}$ of pure foams as a function of volume fraction F. Here, $E_{s}$ is the elastic modulus of the LLDPE powder.

Fig 10. The uniaxial stress-strain relations of ceramic particle reinforced LLDPE foams with $F=0.214$ and $F=0.321$ under quasi-static compression are shown in (a) and (b), respectively. Yield strength $\sigma_{c}$ and elastic modulus $E_{c}$ as a function of volume fraction $f$ are compared between experimental measurements and theoretical predictions obtained from Ruess lower bound (see Appendix B) and analytical models (see Appendix C) are shown in (c) and (d), respectively.

Fig 11. The typical stress-strain curve of LLDPE foams under compression contains elastic region, yield point (Point A), plateau region, onset of densification (Point $B$ ) and densification region.

Fig 12. The absorbed energy per unit volume of particle reinforced foams normalised by the absorbed energy per unit volume of the pure foams, $\widehat{W}_{1}(\varepsilon \leq 0.5,(a))$ and $\widehat{W}_{2}(0.5 \leq \varepsilon \leq 0.8$, (b)), as functions of volume fraction of particle reinforcement $f$ for two selected polymer volume fractions, i.e. $\mathrm{F}=0.214$ and $\mathrm{F}=0.321$.

Fig 13. Comparisons of the normalised absorbed energy $U$ as a function of normalised yield strength $\bar{\sigma}$ among the pure LLDPE foams, the particle reinforced LLDPE foams and a closed-cell elastomeric foam (Gibson and Ashby, 1997). 
Fig 14. Comparisons of engineering stress as a function of engineering strain between the numerical predictions and experimental measurements at selected volume fractions $f$ for (a) $F=0.214,(b) F=0.321$.

Fig 15. The compressive response of the pure LLDPE foam obtained from the FE simulations. (For interpretation of the color legend in this figure, the reader is referred to the web version of this article.)

Fig 16. The compressive response of the particle reinforced LLDPE foam with $\mathrm{F}=0.321, f=0.1$ obtained from the FE simulations. (For interpretation of the color legend in this figure, the reader is referred to the web version of this article.)

Fig 17. The compressive response of the particle reinforced LLDPE foam with $\mathrm{F}=0.321, f=0.2$ obtained from the FE simulations. (For interpretation of the color legend in this figure, the reader is referred to the web version of this article.)

Fig 18. Size effect of particle reinforcement. The functional relations of engineering stress and engineering strain were compared between experimental measurement and simulations with $\mathrm{F}=0.214 f=0.2$. Selected normalised diameters of particles, i.e. $d_{0}=d_{s m} / d_{p m}=0.5,0.588,0.823,1.0$ and 1.176 , were employed in the numerical study to evaluate the size effects of particle reinforcements.

Fig 19. Size effect of the hollow cells. The functional relations of engineering stress and engineering strain were compared between experiments ( $\mathrm{F}=0.214 f=0$ and $\mathrm{F}=0.267 f=0)$ and simulations ( $\mathrm{F}=0.214 f=0)$. The selected normalised diameter of hollow cells, i.e. $D_{0}=D_{d m} / D_{c m}=0.799,1.414,1.712,2.283$ and 2.854 , were employed in the numerical study to evaluate the size effects of hollow cells.

Fig S.1. Creation of the numerical models for pure foams with $F=0.214$. (a) The distributions of cells diameter for the pure foams with $\mathrm{F}=0.214$ and $\mathrm{F}=0.267$ as well as the inserted cells. (b) The hollow cells which follow $D_{c} \sim N\left(D_{c m}=2.9, \gamma_{c m}^{2}=0.25\right)$ were inserted to the foam matrix with 
$F=0.267$ to create the FE model of the pure foam with $F=0.214$. (For interpretation of the color legend in this figure, the reader is referred to the web version of this article.)

Fig S.2. Scale effects of the FE models. The relations of engineering stress and engineering strain of the LLDPE foams with $\mathrm{F}=0.321 f=0, \mathrm{~F}=0.321 f=0.1$ and $\mathrm{F}=0.321 f=0.2$ were compared between simulations obtained from reduced scale FE models with selected values of $\hat{l}$ and experimental measurement. 
Table 1 Characteristics of cells diameters and separations of cells

\begin{tabular}{|c|c|c|c|}
\hline $\begin{array}{l}\text { Volume } \\
\text { fraction of } \\
\text { polymer (F) }\end{array}$ & $\begin{array}{l}\text { Mean diameter } \\
\text { of hollow cells } \\
\qquad D_{c m}(\mathrm{~mm})\end{array}$ & $\begin{array}{l}\text { Variance of hollow } \\
\text { cells diameter } \gamma_{\mathrm{cm}}^{2} \\
\qquad\left(\mathrm{~mm}^{2}\right)\end{array}$ & $\begin{array}{c}\text { Mean } \\
\text { separation } \tilde{e} \\
(\mu \mathrm{m})\end{array}$ \\
\hline 0.214 & 1.752 & 0.642 & 19.31 \\
\hline 0.267 & 1.282 & 0.145 & 36.96 \\
\hline 0.321 & 0.992 & 0.054 & 124.81 \\
\hline 0.428 & 0.572 & 0.014 & 213.36 \\
\hline
\end{tabular}

Table 2 Parameters of brittle cracking

\begin{tabular}{ccccc}
\hline tensile & crack opening & mid-point & mid-point & crack opening \\
strength $\sigma_{t m}$ & $u_{t m}$ & $\psi_{m}$ & $\varepsilon_{m}$ & strain $\varepsilon_{s m}$ \\
\hline $10 \mathrm{MPa}$ & 0.004 & 0.75 & 0.0005 & 0.0005 \\
\hline
\end{tabular}


Table 3: Experimental measurements of yield strength and elastic modulus of LLDPE foams

\begin{tabular}{ccccc}
\hline Foams & $F$ & $f$ & $\begin{array}{c}\text { Yield Strength } \\
(\mathrm{MPa})\end{array}$ & $\begin{array}{c}\text { Elastic } \\
\text { Modulus(MPa) }\end{array}$ \\
\hline Pure foam & 0.214 & 0 & 1.049 & 36.95 \\
& 0.267 & 0 & 1.641 & 44.49 \\
& 0.321 & 0 & 2.146 & 82.79 \\
& 0.428 & 0 & 3.884 & 111.64 \\
\hline Particle reinforced & 0.214 & 0.1 & 1.23 & 42.12 \\
foam (F=0.214) & 0.214 & 0.2 & 1.5 & 63.40 \\
& 0.214 & 0.3 & 2.042 & 98.57 \\
\hline Particle reinforced & 0.321 & 0.1 & 2.92 & 105.37 \\
foam (F=0.321) & 0.321 & 0.2 & 3.44 & 127.63 \\
& 0.321 & 0.3 & 4.367 & 175.35 \\
\hline
\end{tabular}

